\title{
Ehrenfest time in the weak dynamical localization
}

\author{
C. $\operatorname{Tian}^{1}$, A. Kamenev ${ }^{1}$, and A. Larkin ${ }^{1,2,3}$ \\ 1 Department of Physics, University of Minnesota, Minneapolis, MN 55455, USA \\ 2 William I. Fine Theoretical Physics Institute, University of Minnesota, Minneapolis, MN 55455, USA \\ 3 L. D. Landau Institute for Theoretical Physics, Moscow, 117940, Russia
}

(Dated: September 4, 2018)

\begin{abstract}
The quantum kicked rotor (QKR) is known to exhibit dynamical localization in the space of its angular momentum. The present paper is devoted to the systematic first-principal (without a regularizer) diagrammatic calculations of the weak-localization corrections for QKR. Our particular emphasis is on the Ehrenfest time regime - the phenomena characteristic for the classical-to-quantum crossover of classically chaotic systems.
\end{abstract}

PACS numbers: 05.45.-a, 42.50.Vk, 72.15. Rn

\section{INTRODUCTION}

In recent years it became abundantly clear that driven quantum systems exhibit behavior that is qualitatively different from their classical counterparts. Indeed, a driven classically chaotic system exhibits Joel's heating. That is, its average stored energy increases at a steady rate. In other words, such behavior may be characterized as a diffusion in the system's phase space. The remarkable feature of driven quantum systems is finiteness of their phase space motion (localization). Such dynamical localization phenomena was discussed in context of pumped quantum dots 1 , Bose-Einstein condensates subject to pulses of optical standing wave ${ }^{2.3 .4}$, optical microcavity ${ }^{5}$, and other systems.

The simplest model that became a paradigm for studies of the quantum dynamical localization is quantum kicked rotor (QKR). It was numerical discovery of local-

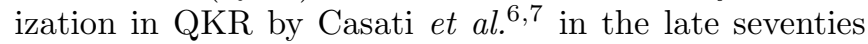
that triggered the broad interest to the subject. Recent progress in trapping of cold atoms and optical manipulation with them lead to experimental realization of the QKR with the unprecedented degree of control2.4. The kicked rotor is described by the time-dependent Hamiltonian:

$$
\hat{H}(t)=\frac{\hat{l}^{2}}{2}+K \cos \hat{\theta} \sum_{n} \delta(t-n)
$$

where angle $\hat{\theta}$ and angular momentum $\hat{l}$ are the pair of canonically conjugated variables. The amplitude of the kicks is described by the dimensionless parameter $K$, also known as the classical stochastically parameter. It is the only parameter of the corresponding classical problem. The quantum problem possesses another dimensionless parameter: the effective Planck constant $k$. The latter enters the problem through the canonical commutation relation: $[\hat{\theta}, \hat{l}]=i \hbar$. The two parameters $K$ and $\hbar$ are straightforwardly related to the optical wavelength, amplitude and atomic mass in cold atoms experiments ${ }^{2.3 .8}$.

Historically, the classical kicked rotor, or standard mapping, first introduced by Chirikov, served as the prototype model for various transport processes in plasmas 9.10 . It was established that the classical dynamics of the kicked rotor exhibits complicated behavior. Most notably it demonstrates the transition from the regular to chaotic motion.10. In particular, for sufficiently large classical parameter $K(\gtrsim 5)$, the chaotic diffusion takes place in the space of angular momentum ${ }^{9.10}$. The latter is associated with the unlimited diffusive expansion of an initially narrow momenta distribution: $\delta\left\langle l^{2}(t)\right\rangle=2 D_{c l} t$. The classical diffusion constant, $D_{c l}$, was a subject of numerous studies ${ }^{9.10}$ and is well understood by now 11.12 . For large stochastically parameter $K \gg 1$, one finds $D_{c l} \approx K^{2} / 4+O\left(K^{3 / 2}\right)$, where the omitted corrections posses an oscillatory dependence on $K$.

The pioneering numerical studies of Casati et al ${ }^{6.7}$ revealed that the corresponding quantum system, $k \neq 0$, behaves in a dramatically different way. The initial diffusive expansion (that is heating) saturates after a certain time, $t_{L}$, and the momentum distribution width stabilizes at $\delta\left\langle l^{2}(t)\right\rangle \sim \xi^{2}=D_{c l} t_{L}$. It was soon suggested in Ref. 13 that similarly to Anderson localization, quantum phase interference may lead to the suppression of classical diffusion for long enough time. This heuristic idea was complemented by mapping QKR onto a one-dimensional tight-binding Anderson model with the pseudo-random potential13.14. Such interpretation leads to the estimate of the localization length as $\xi=D_{c l} / \hbar$ and thus $t_{L}=D_{c l} / \hbar^{2}$. The similarity was further confirmed by studies of a perturbation that breaks the "timereversal symmetry" (TRS) of the QKR ${ }^{15}$. Such perturbation suppresses the survival probability by a factor of two. That is closely analogous to the doubling of the Anderson localization length by the static magnetic field, destroying the interference between a trajectory and its time-reversal partner ${ }^{16.20}$.

If the momentum localization length, $\xi$, is much longer than the "microscopic" scale of the angular momentum (that is $k$ ) then $K \gg k$ and thus $t_{L} \gg 1$. In this case there is a parametrically long crossover regime from the classical diffusion: $\delta\left\langle l^{2}(t)\right\rangle \approx 2 D_{c l} t$ for $1<t \ll t_{L}$ to the strong localization: $\delta\left\langle l^{2}(t)\right\rangle=\xi^{2}$ for $t>t_{L}$. One may be able to develop a systematic perturbation theory in powers of $\left(t / t_{L}\right) \ll 1$, analogous to the weak- 
localization loop expansion in the Anderson localization theory ${ }^{17}$. Such task was undertaken by Altland ${ }^{18}$, who found for the one-loop correction: $\delta\left\langle l^{2}(t)\right\rangle=2 D_{c l} t(1-$ $\left.0.75 \sqrt{t / t_{L}}\right)$. It was suggested furthermore that the universal long-time behavior of the QKR is described by the diffusive supersymmetric nonlinear $\sigma$-model ${ }^{19}$ similar to those employed in the localization theory ${ }^{20}$.

Those calculations essentially map the QKR on a quantum particle in the field of a white-noise random potential. While such analogy is reasonable at long time scales $t \sim t_{L} \gg 1$, it fails to recognize details of the classical to quantum crossover at intermediate time scales. Indeed, a quantized classically chaotic system requires a certain time scale, called the Ehrenfest time, $t_{E}$, to develop quantum interference effects. This fact was realized independently in various contexts 21.22 and now days is well documented in the literature $7,22,23,24,25,26,27$. The physics behind this fact is as follows. To experience the quantum interference, two classical trajectories must converge to a region of the phase space of the size $\delta l \delta \theta \approx k$. Convergence (and divergence) of trajectories in a classically chaotic system is governed by the Lyapunov instability exponent $\lambda$ as e.g. $\delta \theta(t) \sim \exp \{-\lambda t\}$. It thus takes time $t_{E} \sim \lambda^{-1} \ln (1 / k)$ before the interference effects can reveal themselves.

As we show below, for $K \gg 1$ the quantitative definition of the Ehrenfest time for the QKR is given by

$$
t_{E}=\frac{1}{\lambda} \ln \sqrt{\frac{K}{\hbar}},
$$

while the classical Lyapunov exponent is $\lambda=\ln (K / 2)^{9}$. Therefore there is the parametric regime $1 \ll K \ll k^{-1}$, or more precisely:

$$
\ln \left(\frac{1}{\hbar}\right) \gg \ln K>0
$$

where exists a wide separation of the relevant time scales:

$$
1 \ll t_{E} \ll t_{L}
$$

One may expect that such regime is amenable for an analytical treatment of the classical-to-quantum crossover. This problem was first tackled by Aleiner and Larkin ${ }^{27}$ in the context of random classical (long-range) potential scattering (e.g., random Lorentz gases). However, due to the complexity of the Lorentz gas classical dynamics, their treatment required a regularization. The latter is essentially a weak quantum scattering potential added to the Lorentz gas.

The purpose of this paper is to develop a systematic first-principle analytic treatment of the QKR. In particular, we are able to incorporate the semiclassical dynamics at the scale of $t_{E}$ into the weak dynamical localization theory without introducing any regularization. The QKR allows thus to demonstrate explicitly an essential point: existence of the dynamical localization is an intrinsic property of quantized classically chaotic systems - not an artefact of an extraneous regularization. (Remarkably, the Ehrenfest time does not dependend on the regularizer strength ${ }^{28.29 .30}$.) This observation is fully consistent with the early studies of Ehrenfest time ${ }^{21.22}$, suggested the existence of such time scale. For the time interval $t \lesssim t_{L}$, our approach fully encompasses the Ehrenfest regime. The results were reported in the short letter ${ }^{29}$. The main result for the time-dependent spread of the wave-packet may be formulated as:

$$
\delta\left\langle l^{2}(t)\right\rangle=2 D_{c l}\left[t-\frac{4}{3 \sqrt{\pi}} \theta\left(t-4 t_{E}\right) \frac{\left(t-4 t_{E}\right)^{3 / 2}}{t_{L}^{1 / 2}}\right],
$$

where $\theta(t)$ is the standard Heaviside step-function. At intermediate times, $t_{E} \ll t<t_{L}$, Eq. (15) crosses over to the standard weak-localization correction 18 . At shorter times, $t \approx t_{E}$, there is a delay in developing localization given by $4 t_{E}$. A few comments are in due: (i) the actual delay is not absolutely sharp, as suggested by Eq. (5). There are exponentially small deviation from the straight line $2 D_{c l} t$ even for $t<4 t_{E}$, which exact shape is calculated below. (ii) Eq. (5) describes quantum correction linear in $k$ that appears to be delayed by $4 t_{E}$. As first noticed by Shepelansky ${ }^{31}$, quadratic in $\hbar$ corrections show up even at earlier time. However, for at least three first kicks, they may be fully absorbed into a renormalization of the diffusion constant ${ }^{31}\left(\delta D_{c l} \sim k^{2}\right.$, essentially due to the change in the scattering cross-section). It is thus an oversimplification to claim the absence of quantum effects at $t<4 t_{E}$. (iii) Eq. (5) constitutes the one-loop weaklocalization correction. Below we report also the results of the two-loop calculation. It brings the next order correction $\sim \theta\left(t-6 t_{E}\right)\left(t-6 t_{E}\right)^{2} / t_{L}$ that also "protects" the early time evolution from the localization effects. It is still an open problem to sum up the entire series to develop a theory of strong localization that accounts for the Ehrenfest time phenomena.

Most of the existing experiments on atomic gases 3.32 .33 do not fall down in the parametric regime (3), but rather have $k \approx 1$. In this case $t_{E} \approx 1$ and our result, Eq. (5), can only be viewed as a qualitative one. We discuss below other possible realizations of the QKR that utilizes driven Josephson junctions ${ }^{34.35}$. Such systems may prove to be more suitable for exploring the parametric regime (3) and thus for a quantitative comparison with the theory.

The outline of the rest of this paper is as follows: Sec. III is devoted to a qualitative semiclassical picture of the weak-dynamical localizations and the Lyapunov regime. Sections [II [V and V serve to quantify these ideas. In Sec. III the diffusion in the phase space of the kicked rotor is obtained as a classical approximation to the full quantum propagation. Sec. IV is the central part of the present work. It formulates a general framework to deal with the weak dynamical localization at the semiclassical level. In particular, we calculate the frequency-dependent one-loop correction to the classical 
diffusion coefficient and study its effect on the momentum dispersion. This formalism is applied in Sec. $\nabla$ to a modified QKR with broken time-reversal symmetry. The frequency-dependent quantum corrections are calculated at the two-loop level. Experimental realizations of some driven quantum systems are discussed in Sec. VI The effects of noise and dephasing are subject of Sec. VII We conclude in Sec. VIII Some technical details are delegated to Appendices.

\section{QUALITATIVE CONSIDERATIONS}

The physics of the weak-localization corrections is traditionally discussed in the language of classical trajectories. The classical motion of a particle in the random potential is characterized by a rapid randomization of momenta and diffusion spreading of the coordinate. It is thus customary to visualize a trajectory in the coordinate space as a random motion between static impurities. It is straightforward to develop a similar approach for the QKR. In the kicked rotor problem the roles of coordinate and (angular) momentum are interchanged. Indeed, for $K \gg 1$ the angular coordinate $\theta$ is rapidly randomized (over the interval $[-\pi, \pi]$ ), while the angular momentum $l$ acquires a (quasi) random change $\in[-K, K]$. The latter results in the diffusion in the space of angular momentum (see below). We shall thus visualize a "trajectory" as a sequence of values of the angular momentum that kicked rotor "visits" upon successive kicks, Fig. 11

For a quantitative description of the classical motion it is convenient to monitor pairs of angle and angular momentum in discrete moments of time. This maps the classical dynamics onto a so-called standard map:

$$
\begin{aligned}
l_{n+1} & =l_{n}+K \sin \theta_{n} \\
\theta_{n+1} & =\theta_{n}+l_{n+1} .
\end{aligned}
$$

Notice that $l_{n}$ stands for the angular momentum immediately after $(n-1)$-th kick, and $\theta_{n}$ for the angle before $n$-th kick. It is now obvious that the two successive points of the trajectory: $l_{n}$ and $l_{n+1}$ differ by $K \sin \theta_{n}$. As a first approximation one may treat $\theta_{n}$ as uniformly distributed over $[0,2 \pi]$ and thus $\left\langle\left(l_{n+1}-l_{n}\right)^{2}\right\rangle=K^{2}\left\langle\sin ^{2} \theta\right\rangle=K^{2} / 2$. As a result, $\left\langle\left(l_{n}-l_{0}\right)^{2}\right\rangle=2 D_{c l} n$ with $D_{c l}=K^{2} / 4$. An account of the residual correlations between successive $\theta_{n}$ 's, leads to a renormalization of $D_{c l}$ with the next term scaling as $K^{3 / 2}$ etc ${ }^{10.11}$. For completeness of the presentation we shall derive the full result for the classical diffusion constant in Appendix $\mathrm{A}$ For the current qualitative discussion it is enough to appreciate that a trajectory of the classical kicked rotor exhibits random hops in the space of angular momentum, leading to:

$$
\delta\left\langle l^{2}(t)\right\rangle \sim 2 D_{c l} t
$$

This result has a simple physical interpretation: the average energy of the kicked rotor linearly increases with time, reflecting a constant rate Joel's heating. It is exactly this property of the classical kicked rotor that made it useful in the accelerator physics 9.10 .

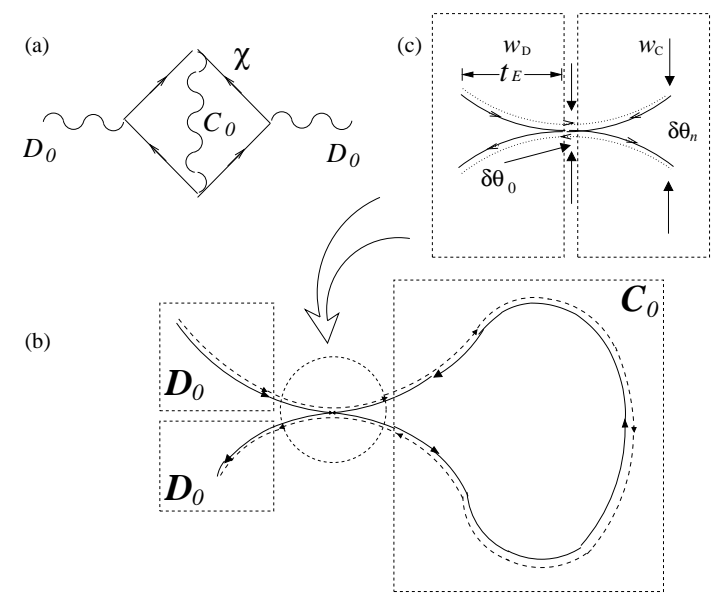

FIG. 1: The first quantum correction to the density-density correlator: (a) one-loop weak localization diagram; (b) trajectory in the momentum space; (c) Hikami box along with Lyapunov portions of the Cooperon and Diffusons.

In the quantum problem an amplitude to evolve from an initial to a finite point in the angular momentum space is given by the sum of amplitudes of all classically allowed trajectories passing through these two points. Generically, different trajectories come with random and uncorrelated phases and thus do not produce a systematic interference contribution. An exception to this rule comes from the trajectories having almost (up to $k$ ) exactly the same geometrical length and thus the same phase. This situation takes place if a trajectory contains a selfintersection point in the angular momentum space. Then another trajectory may exist that is identical to the initial one safe for the direction of propagation along the loop, see Fig. 1 b. The fact that the backward propagation along the loop is consistent with the equations of motion is guaranteed by the time-reversal symmetry of the Hamiltonian:

$$
l \rightarrow l, \quad \theta \rightarrow-\theta, \quad t \rightarrow-t, \quad H \rightarrow H .
$$

(compare it with the time-reversal symmetry in the random potential problem: $\mathbf{r} \rightarrow \mathbf{r}, \mathbf{p} \rightarrow-\mathbf{p}, t \rightarrow-t)$. It is thus easy to see that we are interested in the loops that not only have (almost) coinciding initial and finite momenta: $l_{1} \approx l_{0}$, but also (almost) opposite initial and finite angles: $\theta_{1} \approx-\theta_{0}$. The allowed uncertainty is limited by the effective Planck constant: $\left(l_{1}-l_{0}\right)\left(\theta_{1}+\theta_{0}\right) \lesssim \hbar$.

Since such two trajectories have (almost) the same phase, they interfere constructively and thus lead to a systematic (localization) quantum correction. The probability to complete the loop in time $t$ is called Cooperon and denoted as $\mathcal{C}\left(l_{0}, \theta_{0} ; l_{1}, \theta_{1} ; t\right)$. With the above mentioned conditions on the initial and finite points one may estimate it as $\mathcal{C}(t) \sim \sqrt{D_{c l} / t}$ (this must be multiplied by $k$ to take into account the phase area of the 
allowed uncertainty). This estimate translates (basically by the double integration over time) to the $-k \sqrt{D_{c l}} t^{3 / 2}$ correction 1.18 to the classical law: $\delta\left\langle l^{2}(t)\right\rangle=2 D_{c l} t$. At $t \sim t_{L}=D_{c l} / \hbar^{2}$ the correction exceeds the classical result and the QKR crosses over to the strong localization regime.

The qualitative reasoning given above repeats identically the one employed in the discussion of a particle in the field of the "quantum" white-noise random potential. However, the kicked rotor dynamics possess a very important distinction from that of the white-noise potential problem. The latter is the process without a memory. Indeed, two classical trajectories that identically retrace each other up to a certain point may take completely different roots (in particular counter-propagating ones) after a single "quantum" scattering event. After completing the loop in the opposite directions according to the classical random walk dynamics, another single "quantum" scattering makes the two trajectories to be identical again. These two (actually four, since there are two trajectories involved) quantum scattering events constitute the, so-called, Hikami box ${ }^{36}$, denoted by $\mathcal{X}$ in Fig. 17.

Contrary to this scenario, the kicked rotor scattering events are purely classical, namely the free rotation of the angle. Indeed the trajectory is uniquely defined by the standard map, Eq. (6), sequence. If two trajectories coincide exactly at some point $\left(\theta_{n}, l_{n}\right)$, they continue to be identical (determined by Eq. (6)) forever. This seemingly precludes any possibility to develop the weaklocalization scenario, outlined above. The way out of this apparent paradox is to recall that the loop may be completed not exactly, but rather up to a small uncertainty: $\delta l \delta \theta \lesssim \hbar$. This small initial difference is magnified (more precisely, exponentially increases) upon successive kicking (Lyapunov instability), leading eventually to the two counter-propagating diffusive roots. The situation is rather similar to the localization in the field of the classical large scale random potential (so-called random Lorentz gas). The latter was considered by Aliener and Larkin some time ago 27 . Due to complexity of the Lorentz gas dynamics, they had to introduce quantum impurities (essentially a weak white-noise component of the scattering potential) to treat the problem analytically. The beauty of the QKR is in simplicity of its classical dynamics, Eq. (6), that allows to treat the Lyapunov regime exactly without involving an artificial quantum scattering.

To proceed in this direction, consider two trajectories that initially happen to be at a small distance from each other in the phase space: $(\delta \theta, \delta l)$. Taking variation of Eq. (66), we find that the angle difference evolves as $\delta \theta_{n}=\delta \theta_{n-1}\left(1+K \cos \theta_{n-1}\right)+\delta l_{n-1}$. In the limit $K \gg 1$ it leads to $\delta \theta_{n}=(\delta \theta+\delta l / K) \prod_{k=0}^{n-1}\left(1+K \cos \theta_{k}\right) \simeq$ $(\delta \theta+\delta l / K) e^{\lambda n}$, where $\lambda$ is the Lyapunov exponent. For $K \gg 1$ one finds: $\lambda=\langle\ln (K \cos \theta)\rangle=\ln (K / 2)^{9.10}$ with the residual term $\sim O(1 / K)$. For $\delta \theta \delta l \approx k$, the optimal value of $(\delta \theta+\delta l / K)$ is $\sqrt{\hbar / K}$. It thus takes time
$n=t_{E}=\lambda^{-1} \ln \sqrt{K / \hbar}$, cf. Eq. (2), to evolve from the initial angular uncertainty $\delta \theta \approx \sqrt{\hbar / K} \ll 1$ up to $\delta \theta_{n} \approx 1$, when the diffusive motion takes over. Once this deviation is reached, the usual diffusive spread of the two trajectories takes place. The time-reversal invariance dictates that aforementioned divergence of the two trajectories is preceded by their convergence. The latter takes another $t_{E}$ kicks to be completed. The total duration of the one-way travel through the Lyapunov region is thus $2 t_{E}$. The entire weak-localization loop construction requires two such travels (each including convergence and divergence) through the Lyapunov regime. As a result, the localization corrections are delayed by (can not be developed in time less than) $4 t_{E}$, see Eq. (15).

Technically there are two equivalent ways to incorporate the Lyapunov region into the weak-localization calculations, see Fig. 15. One approach, adopted in Ref. 27, is to redefine Hikami box to contain $4 t_{E}$ scattering events (kicks) instead of conventional four. Then Cooperon (and the Diffusons) is just a conventional diffusive propagator in the momentum space. In the present paper we find convenient to follow the traditional treatment of Hikami box as consisting of the four kicks. These four kicks are treated exactly by multiplying the corresponding quantum evolution operators. It is the analytical expression for Hikami box that dictates allowed deviations of $\delta l \delta \theta$ product. The Lyapunov regimes are delegated to the "legs" of the Cooperon (and Diffusons). The latter now understood as a propagator that includes both Lyapunov-like divergence-convergence of the close trajectories along with the normal diffusion once a macroscopic deviation between them is reached. As we show below, the choice of the four kicks in the Lyapunov regime, that coined to be Hikami box is immaterial. For any such choice the quantum correction, linear in $k$, is delayed by $4 t_{E}$. It is important to mention that the time interval $0<t<4 t_{E}$ is protected from the higher order loop corrections as well. To demonstrate this fact we performed the two-loop weak-localization calculation and found the corresponding contribution $\delta\left\langle l^{2}\right\rangle \sim D_{c l} \theta\left(t-6 t_{E}\right)\left(t-6 t_{E}\right)^{2} / t_{L}$ to be delayed by $6 t_{E}$.

The delay is not absolutely sharp, but rather is slightly smeared by $\delta t_{E} \equiv \sqrt{\lambda_{2} t_{E} / \lambda^{2}} \ll t_{E}$ number of kicks. The reason for this smearing is in fluctuations of the exponent $\lambda$. Such fluctuations are due to the fact that one follows the Lyapunov instability for a finite number of kicks only. (Indeed, unlike classical problems, the minimal deviation is limited by $k$ and thus the time to leave the Lyapunov regime is finite and may fluctuate between the trajectories). Following Ref. 27 we characterize fluctuations of the Lyapunov exponent by the other exponent, $\lambda_{2}$ (for QKR with $K \gg 1$ one finds $\lambda_{2} \approx .82$ ). In the case $t_{E} \gg 1$, cf. Eq. (3), the effect of smearing due to $\lambda_{2}$ is rather small.

The predicted time-dependent momentum dispersion graph is plotted on Fig. 2] The following sections serve to quantify the qualitative semiclassical picture outlined above. 


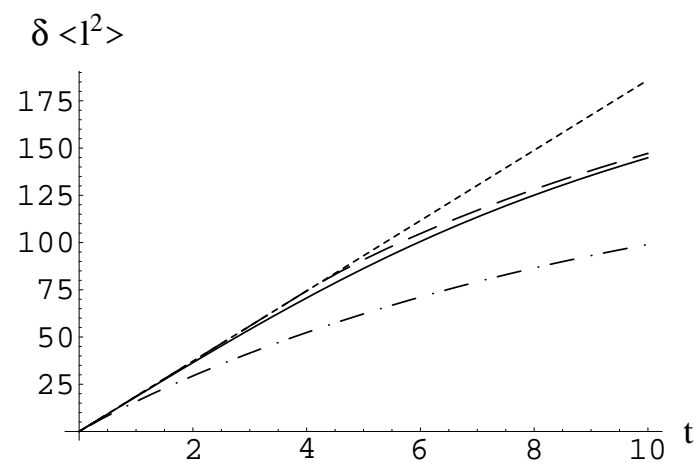

FIG. 2: The momentum dispersion for $K=6.1$ and $\hbar=0.6$ - full line; the classical limit $(k \rightarrow 0)$ - dashed line, standard weak localization $\left(t_{E}=0\right)$ - dashed-dotted line; the limit $\lambda_{2} \rightarrow$ 0, Eq. (5) - long-dashed line.

\section{CLASSICAL LIMIT: DIFFUSON AND COOPERON}

We proceed now to develop the qualitative considerations outlined above into an accurate theory of the QKR. The essential starting point is the classical diffusion in the angular momentum direction of the classical phase space: $[-\pi,+\pi] \times[-\infty,+\infty]$. We'll clarify first how to find the classical diffusion from the exact quantum correlator. One starts from introducing the exact one period quantum evolution operator as:

$$
\begin{aligned}
\hat{U} & \equiv \hat{V} \hat{J} \\
\hat{V} & =\exp \left(i \hat{l}^{2} / 2 \hbar\right), \quad \hat{J}=\exp (i K \cos \hat{\theta} / \hbar) .
\end{aligned}
$$

All physical quantities may be expressed in terms of the matrix elements of $\hat{U}^{n}$, where $n$ stays for the number of kicks (time). We are particularly interested in the fourpoint "density-density" correlator, defined as:

$$
\begin{aligned}
& \mathcal{D}\left(l_{+}, l_{-} ; l_{+}^{\prime}, l_{-}^{\prime} ; n, n^{\prime}\right) \\
\equiv & \left\langle l_{+}\left|\hat{U}^{n} e^{\frac{i \hat{l}^{2}}{2 k}}\right| l_{+}^{\prime}\right\rangle\left\langle l_{-}\left|\hat{U}^{n^{\prime}} e^{\frac{i \hat{l}^{2}}{2 k}}\right| l_{-}^{\prime}\right\rangle^{*}
\end{aligned}
$$

where $\left|l_{ \pm}\right\rangle$denote basis of discrete momentum eigenstates of $K=0$ quantum Hamiltonian. Note that the forward and backward trajectories in this expression are, in general, different. However, it is natural to expect after some transient processes, the correlator will be dominated by the case of $n=n^{\prime}$. This is easy to see by Fourier transforming the correlator with respect to $n, n^{\prime}$, passing to the frequency $\left(\omega_{+}, \omega_{-}\right)$representation:

$$
\begin{aligned}
& \mathcal{D}\left(l_{+}, l_{-} ; l_{+}^{\prime}, l_{-}^{\prime} ; \omega_{+}, \omega_{-}\right) \\
= & \sum_{n, n^{\prime}=0} e^{i\left(\omega_{+} n-\omega_{-} n^{\prime}\right)} \mathcal{D}\left(l_{+}, l_{-} ; l_{+}^{\prime}, l_{-}^{\prime} ; n, n^{\prime}\right)
\end{aligned}
$$

and subsequent averaging over $\left(\omega_{+}+\omega_{-}\right) / 2$. Let us denote such correlator thereby obtained as $\mathcal{D}\left(l_{+}, l_{-} ; l_{+}^{\prime}, l_{-}^{\prime} ; \omega\right)$, where $\omega=\omega_{+}-\omega_{-}$. From Eq. (10) one may check that it satisfies

$$
\begin{aligned}
& \mathcal{D}\left(l_{+}, l_{-} ; l_{+}^{\prime}, l_{-}^{\prime} ; \omega\right)=e^{\frac{i\left(l_{+}^{2}-l_{-}^{2}\right)}{2 \kappa}} \delta_{l_{+}, l_{+}^{\prime}} \delta_{l_{-}, l_{-}^{\prime}} \\
& +e^{i \omega} \sum_{l_{+}^{\prime \prime}, l_{-}^{\prime \prime}}\left\langle l_{+}|\hat{U}| l_{+}^{\prime \prime}\right\rangle\left\langle l_{-}|\hat{U}| l_{-}^{\prime \prime}\right\rangle^{*} \mathcal{D}\left(l_{+}^{\prime \prime}, l_{-}^{\prime \prime} ; l_{+}^{\prime}, l_{-}^{\prime} ; \omega\right)
\end{aligned}
$$

The matrix elements $\left\langle l_{+}|\hat{U}| l_{+}^{\prime \prime}\right\rangle$ and $\left\langle l_{-}|\hat{U}| l_{-}^{\prime \prime}\right\rangle^{*}$ may be explicitly written as

$$
\begin{aligned}
& \left\langle l_{+}|\hat{U}| l_{+}^{\prime \prime}\right\rangle\left\langle l_{-}|\hat{U}| l_{-}^{\prime \prime}\right\rangle^{*} \\
= & \iint \frac{d \theta_{+}}{2 \pi} \frac{d \theta_{-}}{2 \pi} \exp \left[\frac{i l_{+}^{2}}{2 k}+\frac{i K \cos \theta_{+}}{\hbar}+\frac{i \theta_{+}}{k}\left(l_{+}-l_{+}^{\prime \prime}\right)\right] \\
& \times \exp \left[-\frac{i l_{-}^{2}}{2 \hbar}-\frac{i K \cos \theta_{-}}{k}-\frac{i \theta_{-}}{\hbar}\left(l_{-}-l_{-}^{\prime \prime}\right)\right]
\end{aligned}
$$

For what follows, it is convenient to introduce the Wigner transform representation as:

$$
\begin{aligned}
& \mathcal{D}\left(l, \theta ; l^{\prime}, \theta^{\prime} ; \omega\right) \\
\equiv & \sum_{l_{+}-l_{-}} \sum_{l_{+}^{\prime}-l_{-}^{\prime}} \exp \left(-\frac{i}{\hbar}\left[\left(l_{+}-l_{-}\right) \theta-\left(l_{+}^{\prime}-l_{-}^{\prime}\right) \theta^{\prime}\right]\right) \\
& \times \mathcal{D}\left(l_{+}, l_{-} ; l_{+}^{\prime}, l_{-}^{\prime} ; \omega\right)
\end{aligned}
$$

where we define $l \equiv\left(l_{+}+l_{-}\right) / 2$ and $l^{\prime} \equiv\left(l_{+}^{\prime}+l_{-}^{\prime}\right) / 2$.

\section{A. Frobenius-Perron-Ruelle equation: classical kicked rotor}

Let us consider a solution of Eq. (12). As we will see below, the integral over $\theta_{ \pm}$in Eq. (13) are dominated by $\left|\theta_{+}-\theta_{-}\right| \sim \hbar / K \ll 1$. This then allows for a perturbative expansion of $\left|\theta_{+}-\theta_{-}\right|$in the exponent of Eq. (13). In this subsection we show that the leading term in such expansion leads to the classical equation of motion (standard map), Eq. (6) (so-called "semiclassical approximation"). The semiclassical solution thereby obtained is denoted as $\mathcal{D}_{0}$. It should be emphasized that the classical diffusive propagator can not be recovered at this stage. To achieve this goal, the further approximation must be used, which will be clarified in Sec. IIIB

The semiclassical treatment employs the following approximation for $\left\langle l_{+}|\hat{U}| l_{+}^{\prime \prime}\right\rangle\left\langle l_{-}|\hat{U}| l_{-}^{\prime \prime}\right\rangle^{*}$ matrix elements, cf. Eq. (13), 


$$
\begin{aligned}
& \left\langle l_{+}|\hat{U}| l_{+}^{\prime \prime}\right\rangle\left\langle l_{-}|\hat{U}| l_{-}^{\prime \prime}\right\rangle^{*} \approx \iint \frac{d \theta_{+}}{2 \pi} \frac{d \theta_{-}}{2 \pi} \exp \left[\frac{i\left(l_{+}+l_{-}\right)\left(l_{+}-l_{-}\right)}{2 \hbar}-\frac{i K}{\hbar} \sin \frac{\theta_{+}+\theta_{-}}{2}\left(\theta_{+}-\theta_{-}\right)\right] \\
\times & \exp \left\{\frac{i}{\hbar}\left(\theta_{+}-\theta_{-}\right)\left(\frac{l_{+}+l_{-}}{2}-\frac{l_{+}^{\prime \prime}+l_{-}^{\prime \prime}}{2}\right)+\frac{i}{\hbar} \frac{\theta_{+}+\theta_{-}}{2}\left[\left(l_{+}-l_{-}\right)-\left(l_{+}^{\prime \prime}-l_{-}^{\prime \prime}\right)\right]\right\} .
\end{aligned}
$$

Let us insert it into Eq. (12) and perform the Wigner transform. We also define $\theta \equiv\left(\theta_{+}+\theta_{-}\right) / 2$ and $\theta^{\prime} \equiv\left(\theta_{+}^{\prime}+\right.$ $\left.\theta_{-}^{\prime}\right) / 2$ to simplify the notations. Then with $\left(\theta_{+}-\theta_{-}\right) / \hbar$, $\left(l_{+}-l_{-}\right) / \hbar,\left(l_{+}^{\prime}-l_{-}^{\prime}\right) / \hbar$ and $\left(l_{+}^{\prime \prime}-l_{-}^{\prime \prime}\right) / \hbar$ integrated out, we obtain:

$$
\begin{aligned}
\mathcal{D}_{0}\left(l, \theta ; l^{\prime}, \theta^{\prime} ; \omega\right)= & 2 \pi \hbar \delta\left(l-l^{\prime}\right) \delta\left(\theta-\theta^{\prime}-l\right) \\
& +e^{i \omega} \vec{P} \mathcal{D}_{0}\left(l, \theta ; l^{\prime}, \theta^{\prime} ; \omega\right),
\end{aligned}
$$

where $\vec{P}$ is the Frobenius-Perron-Ruelle (FPR) operator, acting on the nearest two arguments from the left according to:

$$
\begin{aligned}
& \vec{P} f\left(l, \theta ; l^{\prime}, \theta^{\prime}\right) \\
\equiv & \iint d l_{1} d \theta_{1} \delta\left(l-l_{1}-K \sin \theta_{1}\right) \delta\left(\theta-\theta_{1}-l\right) \\
& \times f\left(l_{1}, \theta_{1} ; l^{\prime}, \theta^{\prime}\right),
\end{aligned}
$$

where $f\left(l, \theta ; l^{\prime}, \theta^{\prime}\right)$ is an arbitrary function. The kernel above implies that the correlator thereby obtained describes the deterministic motion of classical kicked rotor, i.e., standard mapping. Note, that in the time representation, $\mathcal{D}_{0}$ is normalized, namely $(2 \pi k)^{-1} \iint d l d \theta \mathcal{D}_{0}\left(l, \theta ; l^{\prime}, \theta^{\prime} ; n\right)=1$.

\section{B. Diffusion approximation}

To recover the classical diffusion the procedure of deriving FPR equation must be appropriately regularized 11.12 .37 . Indeed, in the presence of noises one is able to do so and find the proper diffusion constant. We shall not follow this procedure here, but rather refer a reader to Refs. 11 12.

We shall show below, however, that a regularization is consistent with the generalization of Altland's $\frac{18}{}$ diagrammatic method. The latter starts from exact quantum density-density correlator, Eq. (10), and leads to the classical diffusion with the correct diffusion constant. The basic idea is that for sufficiently large $K$, there must be some time scale, say $\tau_{c}$, beyond which any classical trajectory loses memory about its initial conditions. Technically, complementing the semiclassical approximation: $\left|\theta_{n_{+}}-\theta_{n_{-}}\right| \ll 1, n=1,2, \cdots$, it is further required that $l_{+}=l_{-} \equiv l$ and $l_{+}^{\prime}=l_{-}^{\prime} \equiv l^{\prime}$ at two ends, as well as $l_{+}^{\prime \prime}=l_{-}^{\prime \prime} \equiv l^{\prime \prime}$ for the intermediate variables at multiple times of $\tau_{c}$.

For $\tau_{c}=1$ the angular memory is lost after every kick (cf. the first diagram on the right hand side of Fig. 3 b). (a)

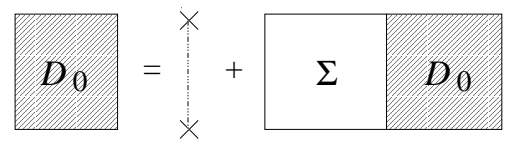

(b)

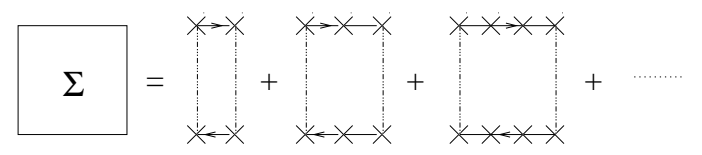

$$
\begin{aligned}
\times=1 & \times \vec{V} \text { or } \hat{V}^{+} \\
\times & \rightarrow=\hat{J} \quad \leftarrow=\hat{J}^{+}
\end{aligned}
$$

FIG. 3: Schematic representation for the diffusion approximation of classical density-density correlator - the solution of Frobenius-Perron-Ruelle equation (a), with the self-energy (b). The structure of the self-energy is such that the beginning and ending (after $\tau_{c}$ kicks) pairs of the angular momenta are the same (denoted by vertical dot-dashed line), while the other pairs not.

In this approximation Eq. (12) is reduced to

$$
\begin{aligned}
& \mathcal{D}_{0}\left(l, l^{\prime} ; \omega\right) \\
= & \delta_{l, l^{\prime}}+e^{i \omega} \sum_{l^{\prime \prime}}\left\langle l|\hat{U}| l^{\prime \prime}\right\rangle\left\langle l|\hat{U}| l^{\prime \prime}\right\rangle^{*} \mathcal{D}_{0}\left(l^{\prime \prime}, l^{\prime} ; \omega\right),
\end{aligned}
$$

where the matrix element $\left\langle l|\hat{U}| l^{\prime \prime}\right\rangle\left\langle l|\hat{U}| l^{\prime \prime}\right\rangle^{*}$ is explicitly written as

$$
\begin{aligned}
& \left\langle l|\hat{U}| l^{\prime \prime}\right\rangle\left\langle l|\hat{U}| l^{\prime \prime}\right\rangle^{*}=\left\langle l|\hat{J}| l^{\prime \prime}\right\rangle\left\langle l|\hat{J}| l^{\prime \prime}\right\rangle^{*} \\
= & \iint \frac{d \theta_{+}}{2 \pi} \frac{d \theta_{-}}{2 \pi} \exp \left[\frac{i K}{\hbar}\left(\cos \theta_{+}-\cos \theta_{-}\right)\right] \\
& \times \exp \left[\frac{i\left(\theta_{+}-\theta_{-}\right)\left(l-l^{\prime \prime}\right)}{\hbar}\right] \\
= & \iint \frac{d \theta_{+}}{2 \pi} \frac{d \theta_{-}}{2 \pi} \exp \left[-\frac{2 i K}{\hbar} \sin \frac{\theta_{+}+\theta_{-}}{2} \sin \frac{\theta_{+}-\theta_{-}}{2}\right] \\
& \times \exp \left[\frac{i\left(\theta_{+}-\theta_{-}\right)\left(l-l^{\prime \prime}\right)}{\hbar}\right] .
\end{aligned}
$$

From this we see (as mentioned above) that the integral is dominated by $\left|\theta_{+}-\theta_{-}\right| \sim \hbar / K$. Making the change of variables: $\left(\theta_{+}, \theta_{-}\right) \rightarrow\left[\left(\theta_{+}+\theta_{-}\right) / 2, \theta_{+}-\theta_{-} \equiv k \varphi\right]$ and 
integrating out $\left(\theta_{+}+\theta_{-}\right) / 2$, we simplify Eq. (19) as ${ }^{18}$

$$
\left\langle l|\hat{U}| l^{\prime \prime}\right\rangle\left\langle l|\hat{U}| l^{\prime \prime}\right\rangle^{*} \approx \int \frac{d \varphi}{2 \pi} J_{0}\left[\frac{2 K}{\hbar} \sin \frac{\hbar \varphi}{2}\right] e^{i \varphi\left(l-l^{\prime \prime}\right)} .
$$

Here $J_{n}(x)$ is the Bessel function of order $n$. Turning to the Fourier representation: $\mathcal{D}_{0}(\varphi ; \omega) \equiv$ $\sum_{l-l^{\prime}} e^{-i \varphi\left(l-l^{\prime}\right)} \mathcal{D}_{0}\left(l-l^{\prime} ; \omega\right)$, Eq. (18) gives the classical propagator as

$$
\mathcal{D}_{0}(\varphi ; \omega)=\frac{1}{1-e^{i \omega} J_{0}\left[\frac{2 K}{\hbar} \sin \frac{\hbar \varphi}{2}\right]} .
$$

In the limit $\omega \ll 1, K \varphi \ll 1$, it is reduced to the usual diffuson (Fig. [3):

$$
\mathcal{D}_{0}(\varphi ; \omega) \approx \frac{1}{-i \omega+D_{c l} \varphi^{2}}
$$

with the diffusion constant $D_{c l}(K)=K^{2} / 4$.

In Ref. 11, it was shown that the higher order correlations, namely $\tau_{c}>1$ [e.g., the second, third, etc. diagrams on the right hand side of Fig. [3 $\mathrm{b}$ ] lead to the modification of the diffusion constant according to

$$
D_{c l}(K)=\frac{K^{2}}{4}\left[1-2 J_{2}(K)-2 J_{1}^{2}(K)+2 J_{2}^{2}(K)+\cdots\right] \text {. }
$$

We should emphasize that although the original derivation is based on pure classical considerations, it is fully compatible with the general formalism developed in this paper. For the clarification, we reproduce Eq. (23) from the exact quantum density-density correlator, Eq. (12), in Appendix A

One may wonder whether the dynamics of a classical system may be indeed described by the diffusion in the long time limit. The answer is known to depend on the initial conditions. In particular, it is well-known that for any $K$, there exist stable islands in the phase space, where a trajectory exhibits quasi-periodic motion 9,10 . It has been estimated, however, that the total area of these islands on the phase space is exponentially small in the limit $K \gg 1^{9,23}$. Our approximation, being an expansion in powers of $1 / K$, is bound to lose information about these islands. It has also been known that for some parametric regions of $K$, there are some peculiar islands (so-called "accelerator mode" 9.10 ), starting from or near which a trajectory will be boosted, faster than the normal diffusion 38 . (Similar phenomenon exist also in a generic mode10.32 . ) Throughout the paper we will stay away from these parametric regions, and focus on the large $K$ case with the assumption that the initial conditions do not fall neither into the stable islands nor to the accelerator modes.

\section{Classical Cooperon}

Starting from the exact quantum density-density correlator, one may see that the classical diffuson is essentially related to the limiting case: $\left|\theta_{1+}-\theta_{1-}\right| \sim$ $\left|\theta_{2+}-\theta_{2-}\right| \cdots \sim\left|\theta_{n+}-\theta_{n-}\right| \ll 1$. In this part, we turn to the discussion of another important limiting case, i.e., $\left|\theta_{1+}+\theta_{n-}\right| \sim\left|\theta_{2+}+\theta_{(n-1)-}\right| \cdots \sim\left|\theta_{n+}+\theta_{1-}\right| \ll 1$ (cf. Fig. 4 for notations).

\section{Diffusion approximation of the solution of FPR equation}

To find the density-density correlator in this limit let us pass to Wigner representation (notice the crucial difference with Eq. (14)):

$$
\begin{aligned}
& \mathcal{C}\left(l, \theta ; l^{\prime}, \theta^{\prime} ; \omega\right) \\
\equiv & \sum_{l_{+}-l_{-}^{\prime}} \sum_{l_{+}^{\prime}-l_{-}} \exp \left(-\frac{i}{\hbar}\left[\left(l_{+}-l_{-}^{\prime}\right) \theta-\left(l_{+}^{\prime}-l_{-}\right) \theta^{\prime}\right]\right) \\
& \times \mathcal{C}\left(l_{+}, l_{-} ; l_{+}^{\prime}, l_{-}^{\prime} ; \omega\right)
\end{aligned}
$$

where we define $l \equiv\left(l_{+}+l_{-}^{\prime}\right) / 2$ and $l^{\prime} \equiv\left(l_{+}^{\prime}+l_{-}\right) / 2$. The key observation is that the diffuson diagram may be retrieved with the bottom (advanced Green function) line [Fig. 4 (a)] rotated. This is due to the fact that such a procedure simply leads to the time-reversal of the series: $\theta_{1-} \rightarrow \theta_{2-} \rightarrow \cdots \rightarrow \theta_{n-}$ such that it becomes $-\theta_{n-} \rightarrow-\theta_{n-1-} \rightarrow \cdots \rightarrow-\theta_{1-}$. For this reason, we call it Cooperon as introduced in Sec. III Since $\left|\theta_{1+}+\theta_{n-}\right| \ll$ 1, we may proceed along the lines of derivation of Eq. (16) from Eqs. (12) and (14), and arrive at

$$
\begin{aligned}
\mathcal{C}_{0}\left(l, \theta ; l^{\prime}, \theta^{\prime} ; \omega\right)= & 2 \pi \hbar \delta\left(l-l^{\prime}\right) \delta\left(\theta-\theta^{\prime}-l\right) \\
& +e^{i \omega} \vec{P} \mathcal{C}_{0}\left(l, \theta ; l^{\prime}, \theta^{\prime} ; \omega\right) .
\end{aligned}
$$

That is, the classical Cooperon is also a solution of FPR equation.

(a)

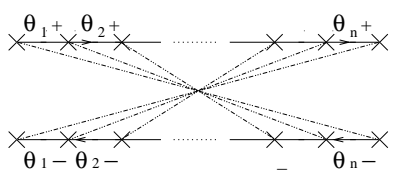

(b)

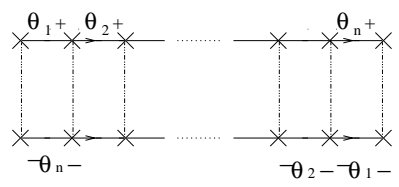

FIG. 4: The diffusive Cooperon approximation for the classical density-density correlator. Typical diagrammatic representation for the classical Cooperon $\left(\left|\theta_{1+}+\theta_{n-}\right|, \mid \theta_{2+}+\right.$ $\left.\theta_{(n-1)-} \mid, \cdots \ll 1\right)$ (a). Rotating the bottom line of (a) retrieves classical diffuson with $\theta_{k-}$ replaced by $-\theta_{k-}(k=$ $1,2, \cdots, n)(\mathrm{b})$.

The only difference with the Diffuson is that instead of having poles at $k \varphi \equiv \theta_{+}-\theta_{-}$, the Cooperon has poles at $k \varphi \equiv \theta_{+}+\theta_{-}$(i.e., $k \varphi \equiv \theta_{1+}+\theta_{n-}=\theta_{2+}+\theta_{(n-1)-}=$ 
$\left.\cdots=\theta_{n+}+\theta_{1-}\right)$. One concludes, thus, that the diffusive form, Eq. (22), holds in the limit $\omega \ll 1, K \varphi \ll 1$ (cf. Fig. 4) for the Cooperon as well. In particular, in the case of $\left|\theta+\theta^{\prime}\right| \sim 1$, the averaging over $\left(\theta+\theta^{\prime}\right) / 2$ may be performed. Furthermore, if $\left|l-l^{\prime}\right| \lesssim K$, then $\mathcal{C}_{0}$ does not depend on the angular momenta. In other words,

$$
\begin{aligned}
& \mathcal{C}_{0}\left(l, \theta ; l^{\prime},-\theta^{\prime} ; \omega\right)=\left\langle\mathcal{C}_{0}\left(l, \theta ; l^{\prime},-\theta^{\prime} ; \omega\right)\right\rangle_{\left(\theta+\theta^{\prime}\right) / 2} \\
& =\int \frac{d \varphi}{2 \pi} \frac{\hbar}{-i \omega+D_{c l} \varphi^{2}} \equiv\left\langle\mathcal{C}_{0}(\omega)\right\rangle,
\end{aligned}
$$

where $\left|l-l^{\prime}\right| \lesssim K$, and $\left|\theta-\theta^{\prime}\right| \sim 1$.

\section{Treatment of the Lyapunov instability regime}

The above general solution for the classical Cooperon, $\mathcal{C}_{0}\left(l, \theta ; l^{\prime},-\theta^{\prime} ; n\right)$, characterizes the probability for a trajectory, initiating from $(l, \theta)$, to end at $\left(l^{\prime},-\theta^{\prime}\right)$. From now on we focus on a special case, where $\delta l_{0} \equiv l-$ $l^{\prime} ; \delta \theta_{0} \equiv \theta-\theta^{\prime}$ are such small that $\left|\delta l_{0}\right| \ll K,\left|\delta \theta_{0}\right| \ll 1$. In this part we show that it differs from $\left\langle\mathcal{C}_{0}(\omega)\right\rangle$ by a renormalization factor.

Without loss of generality, we assume that $\mathcal{C}_{0}\left(l, \theta ; l^{\prime},-\theta^{\prime} ; n\right)$ evolves from some initial distribution $f\left(l, \theta ; l^{\prime},-\theta^{\prime}\right)$, bearing the symmetry of $f\left(l, \theta ; l^{\prime},-\theta^{\prime}\right)=f\left(l^{\prime}, \theta^{\prime} ; l,-\theta\right)$. Then the formal solution of the FPR equation is given by

$$
\mathcal{C}_{0}\left(l, \theta ; l^{\prime},-\theta^{\prime} ; n\right)=\vec{P}^{n} f\left(l, \theta ; l^{\prime},-\theta^{\prime}\right) .
$$

To simplify the notations, we rewrite the action of FPR operator on $\mathcal{C}_{0}$ as:

$$
\vec{P} \mathcal{C}_{0}\left(l, \theta ; l^{\prime}, \theta^{\prime}\right)=\mathcal{C}_{0}\left(\mathcal{S}^{-1}[l, \theta] ; l^{\prime}, \theta^{\prime}\right),
$$

where $\mathcal{S}$ and $\mathcal{S}^{-1}$ are defined as

$$
\begin{aligned}
\mathcal{S}[l, \theta] & \equiv(l+K \sin (\theta+l), \theta+l), \\
\mathcal{S}^{-1}[l, \theta] & \equiv(l-K \sin (\theta-l), \theta-l),
\end{aligned}
$$

respectively. Moreover, we introduce the time-reversal of FPR operator, $\overleftarrow{P}_{T}$, as

$$
\begin{array}{r}
f\left(l, \theta ; l^{\prime}, \theta^{\prime}\right) \overleftarrow{P}_{T} \equiv \iint d l_{1} d \theta_{1} f\left(l, \theta ; l_{1}, \theta_{1}\right) \\
\times \delta\left(l_{1}-l^{\prime}-K \sin \theta_{1}\right) \delta\left(\theta_{1}-\theta^{\prime}-l\right)
\end{array}
$$

Owing to the symmetry of $f$, mentioned above, one may introduce the following identity:

$$
\vec{P} f\left(l, \theta ; l^{\prime}, \theta^{\prime}\right)=f\left(l, \theta ; l^{\prime}, \theta^{\prime}\right) \overleftarrow{P}_{T}
$$

its proof is given in Appendix [B] Applying repeatedly this relation to the formal solution, Eq. (27), we obtain:

$$
\begin{aligned}
& \mathcal{C}_{0}\left(l, \theta ; l^{\prime},-\theta^{\prime} ; n\right) \\
= & \vec{P}^{n-2 n^{\prime}}\left\{\vec{P}^{n^{\prime}} f\left(l, \theta ; l^{\prime},-\theta^{\prime}\right) \overleftarrow{P}_{T}^{n^{\prime}}\right\} \\
= & \vec{P}^{n-2 n^{\prime}} f\left(\mathcal{S}^{-n^{\prime}}[l, \theta] ; \mathcal{S}^{n^{\prime}}\left[l^{\prime},-\theta^{\prime}\right]\right)
\end{aligned}
$$

for an arbitrary integer number $n^{\prime}$ such that $2 n^{\prime} \leq n$. Consider two nearby trajectories described by $\left(l_{1}, \theta_{1}\right)$ and $\left(l_{1}^{\prime}, \theta_{1}^{\prime}\right)$, respectively. Their motion is induced by $\mathcal{S}^{-n}[l, \theta]$ and $\mathcal{S}^{n}\left[l^{\prime},-\theta^{\prime}\right]$ following

$$
\begin{aligned}
\left(l_{1}, \theta_{1}\right) & \equiv \mathcal{S}^{-n}[l, \theta], \\
\left(l_{1}^{\prime},-\theta_{1}^{\prime}\right) & \equiv \mathcal{S}^{n}\left[l^{\prime},-\theta^{\prime}\right] .
\end{aligned}
$$

Associated with the exponential separation of these two nearby trajectories, the time, say $n_{\mathrm{c}}$, is defined such that

$$
\left|l_{n_{c}}-l_{n_{c}}^{\prime}\right| \approx K, \quad\left|\theta_{n_{c}}-\theta_{n_{c}}^{\prime}\right| \approx 1
$$

At such a moment the separation reaches some macroscopic size. After this time the separation experiences the usual diffusion in the angular momentum space, while the angle difference is uniformly distributed. We substitute then $n^{\prime}=n_{\mathrm{c}}$ into Eq. (32), and arrive at

$$
\mathcal{C}_{0}\left(l, \theta ; l^{\prime},-\theta^{\prime} ; n\right)=\theta\left(n-2 n_{\mathrm{c}}\right)\left\langle\mathcal{C}_{0}\left(n-2 n_{\mathrm{c}}\right)\right\rangle
$$

where $\left\langle\mathcal{C}_{0}(n)\right\rangle$ is the Fourier transform of $\left\langle\mathcal{C}_{0}(\omega)\right\rangle$. Consequently, the Fourier transform of Eq. (32) with respect to $n$ is reduced to

$$
\mathcal{C}_{0}\left(l, \theta ; l^{\prime},-\theta^{\prime} ; \omega\right)=e^{2 i \omega n_{\mathrm{c}}} \int \frac{d \varphi}{2 \pi} \frac{\hbar}{-i \omega+D_{c l} \varphi^{2}}
$$

In general, $n_{\mathrm{c}}$ is determined by the initial condition, namely the center of mass: $\left[\left(l+l^{\prime}\right) / 2,\left(\theta+\theta^{\prime}\right) / 2\right]$ and the initial deviation: $(\delta l, \delta \theta)$. One can perform the change of variables with respect to general separation $(\delta l, \delta \theta)$ according to $z \equiv \ln |\delta \theta|, \alpha \equiv \delta l / \delta \theta$. In what follows $z$ and $\alpha$ are identified as slow and fast variables, correspondingly. If the initial deviation is small enough such that the typical $n_{\mathrm{c}} \gg 1$, the fluctuations of $n_{\mathrm{c}}$ at this time scale are small. As a result, after averaging over initial $\alpha$, as well as the center of mass, one may cast $\exp \left[2 i \omega n_{\mathrm{c}}\right]$ into the renormalization factor (with the logarithmic accuracy) as (see Appendix C for details)

$$
\begin{aligned}
\mathcal{W}_{\mathrm{C}}(2 \omega) & \equiv\left\langle\exp \left[2 i \omega n_{\mathrm{c}}\right]\right\rangle \\
& =\exp \left(2 i \omega t^{\mathrm{C}}-\frac{2 \omega^{2} \lambda_{2} t^{\mathrm{C}}}{\lambda^{2}}\right)
\end{aligned}
$$

with

$$
t^{\mathrm{C}} \equiv \frac{1}{\lambda}\left|\ln \frac{1}{\sqrt{\delta \theta^{2}+\delta l^{2} / K^{2}}}\right|
$$

in the limit $\omega \lambda_{2} / \lambda^{2} \ll 1$. Consequently, Eq. (36) is reduced to

$$
\mathcal{C}_{0}\left(l, \theta ; l^{\prime},-\theta^{\prime} ; \omega\right)=\hbar \mathcal{W}_{\mathrm{C}}(2 \omega) \int \frac{d \varphi}{2 \pi} \frac{1}{-i \omega+D_{c l} \varphi^{2}}
$$




\section{WEAK DYNAMICAL LOCALIZATION IN KICKED ROTOR: ONE-LOOP CORRECTION}

As explained above the weak dynamical localization involves couplings between the Diffusons and the Cooperon [Fig. [5. Therefore one needs a technique to treat two different kinds of the Wigner transforms introduced for Diffusons, Eq. (14), and for Cooperons, Eq. (24), in a unified way. To develop such a technique is the central task of this section. We show then that the constructive interference between two counter-propagating trajectories leads to the usual one-loop quantum correction, which is a precursor of the dynamical localization. In particular, the one-loop correction to the diffusion constant will be calculated.

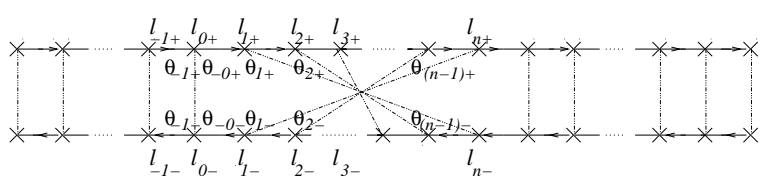

FIG. 5: Sketch of a general diagram, leading to one-loop approximation.

\section{A. Exact interaction vertex}

We will show in Appendix D1 that the one-loop correction to the density-density correlator reads as

$$
\delta \hat{\mathcal{D}}=\hat{\mathcal{D}}\left(e^{i \omega} \hat{\mathcal{P}}_{T}-1\right) \hat{\mathrm{C}}\left(e^{i \omega} \hat{\mathcal{P}}-1\right) \hat{\mathcal{D}}
$$

with

$$
\hat{\mathrm{C}} \equiv e^{i \omega} \hat{\mathcal{P}}_{J} \hat{\mathcal{C}}_{0} e^{i \omega} \hat{\mathcal{P}}_{J}
$$

in the exact quantum operator representation. Here $\hat{\mathcal{P}} \equiv$ $\hat{\mathcal{P}}_{V} \hat{\mathcal{P}}_{J}$ and $\hat{\mathcal{P}}_{T} \equiv \hat{\mathcal{P}}_{J} \hat{\mathcal{P}}_{V}$. The matrix elements of $\hat{\mathcal{P}}_{V}$ and $\hat{\mathcal{P}}_{J}$ are explicitly written as

$$
\begin{aligned}
\left\langle l_{+}, l_{-}\left|\hat{\mathcal{P}}_{V}\right| l_{+}^{\prime}, l_{-}^{\prime}\right\rangle= & \exp \left[\frac{i\left(l_{+}^{2}-l_{-}^{2}\right)}{2 k}\right] \delta_{l_{+}, l_{+}^{\prime}} \delta_{l_{-}, l_{-}^{\prime}}, \\
\left\langle\theta_{+}, \theta_{-}\left|\hat{\mathcal{P}}_{J}\right| \theta_{+}^{\prime}, \theta_{-}^{\prime}\right\rangle= & \exp \left[\frac{i K\left(\cos \theta_{+}-\cos \theta_{-}\right)}{\hbar}\right] \\
& \times \delta\left(\theta_{+}-\theta_{+}^{\prime}\right) \delta\left(\theta_{-}-\theta_{-}^{\prime}\right)
\end{aligned}
$$

in the representation of the angular momentum and the angle, correspondingly. Since we are ultimately interested in the long time effects and therefore in the low frequencies the $e^{i \omega}$ factors in Eqs. (40) and (41) may be safely omitted from now on. Thus, we shall not write them hereafter.

\section{Minimal wave packet}

In order to calculate $\delta \hat{\mathcal{D}}$ explicitly, we consider a general quantity, say $\hat{\mathcal{I}}_{q} \equiv \hat{\mathcal{A}} \hat{\mathrm{C}} \hat{\mathcal{B}}$ (understanding that $\hat{\mathcal{A}}=$ $\hat{\mathcal{D}}\left(\hat{\mathcal{P}}_{T}-1\right), \hat{\mathcal{B}}=(\hat{\mathcal{P}}-1) \hat{\mathcal{D}}$ for $\left.\delta \hat{\mathcal{D}}\right)$, and write it explicitly as (for simplicity we omit $\omega$ argument)

$$
\begin{aligned}
& \mathcal{I}_{q}\left(l_{+}, l_{-} ; l_{+}^{\prime}, l_{-}^{\prime}\right)=\sum_{l_{1}, l_{1}^{\prime} l_{2}, l_{2}^{\prime} l_{3}, l_{3}^{\prime} l_{4}, l_{4}^{\prime}} \sum_{\times \mathcal{A}\left(l_{+}, l_{-} ; l_{1}, l_{1}^{\prime}\right) \mathcal{X} \mathcal{C}_{0}\left(l_{2}, l_{3} ; l_{2}^{\prime}, l_{3}^{\prime}\right) \mathcal{B}\left(l_{4}, l_{4}^{\prime} ; l_{+}^{\prime}, l_{-}^{\prime}\right)}
\end{aligned}
$$

in the angular momentum representation, where $\mathcal{X}$ is

$$
\begin{aligned}
\mathcal{X}= & \left\langle l_{1}\left|e^{i \frac{K}{k} \cos \hat{\theta}}\right| l_{2}\right\rangle\left\langle l_{1}^{\prime}\left|e^{i \frac{K}{k} \cos \hat{\theta}}\right| l_{2}^{\prime}\right\rangle^{*} \\
& \times\left\langle l_{3}\left|e^{i \frac{K}{K} \cos \hat{\theta}}\right| l_{4}\right\rangle\left\langle l_{3}^{\prime}\left|e^{i \frac{K}{\hbar} \cos \hat{\theta}}\right| l_{4}^{\prime}\right\rangle^{*} .
\end{aligned}
$$

Furthermore, we write down explicitly the matrix elements of $\mathcal{X}$, Eq. (44), as

$$
\mathcal{X}=\iiint \int \frac{d \theta_{1}}{2 \pi} \frac{d \theta_{1}^{\prime}}{2 \pi} \frac{d \theta_{2}}{2 \pi} \frac{d \theta_{2}^{\prime}}{2 \pi} \exp \left[\frac{i}{\hbar}\left(S_{k i n}+S_{p}\right)\right],
$$

where $S_{k i n}$ and $S_{p}$ are defined as

$$
\begin{aligned}
S_{k i n}= & -\theta_{1}\left(l_{1}-l_{2}\right)+\theta_{1}^{\prime}\left(l_{1}^{\prime}-l_{2}^{\prime}\right) \\
& -\theta_{2}\left(l_{3}-l_{4}\right)+\theta_{2}^{\prime}\left(l_{3}^{\prime}-l_{4}^{\prime}\right)
\end{aligned}
$$

and

$$
S_{p}=K\left(\cos \theta_{1}-\cos \theta_{1}^{\prime}+\cos \theta_{2}-\cos \theta_{2}^{\prime}\right),
$$

respectively. For the discussions below, we make the following change of variables:

$$
\begin{aligned}
& \phi=\left(\theta_{1}-\theta_{1}^{\prime}\right)-\left(\theta_{2}-\theta_{2}^{\prime}\right), \\
& \delta l_{1}=\frac{l_{2}+l_{3}^{\prime}}{2}-\frac{l_{3}+l_{2}^{\prime}}{2}, \quad \delta \theta_{1}=\frac{\theta_{1}+\theta_{1}^{\prime}}{2}+\frac{\theta_{2}+\theta_{2}^{\prime}}{2} \\
& \delta l_{2}=\frac{l_{1}+l_{1}^{\prime}}{2}-\frac{l_{4}+l_{4}^{\prime}}{2} \\
& \delta \theta_{2}=-\frac{1}{2}\left[\left(\theta_{1}-\theta_{1}^{\prime}\right)+\left(\theta_{2}-\theta_{2}^{\prime}\right)\right] .
\end{aligned}
$$

Then $S_{k i n}$ may be rewritten as: 


$$
\begin{aligned}
S_{k i n}= & \frac{\phi}{2}\left[\left(\frac{l_{2}+l_{3}^{\prime}}{2}+\frac{l_{3}+l_{2}^{\prime}}{2}\right)-\left(\frac{l_{1}+l_{1}^{\prime}}{2}+\frac{l_{4}+l_{4}^{\prime}}{2}\right)\right]+\delta \theta_{1} \delta l_{1}+\delta \theta_{2} \delta l_{2} \\
& -\frac{1}{2}\left(\frac{\theta_{1}+\theta_{1}^{\prime}}{2}-\frac{\theta_{2}+\theta_{2}^{\prime}}{2}\right)\left\{\left[\left(l_{1}-l_{1}^{\prime}\right)+\left(l_{4}-l_{4}^{\prime}\right)\right]-\left[\left(l_{2}-l_{3}^{\prime}\right)+\left(l_{3}-l_{2}^{\prime}\right)\right]\right\} \\
& -\frac{\delta \theta_{1}}{2}\left[\left(l_{1}-l_{1}^{\prime}\right)-\left(l_{4}-l_{4}^{\prime}\right)\right]-\frac{\delta \theta_{2}}{2}\left[\left(l_{2}-l_{3}^{\prime}\right)-\left(l_{3}-l_{2}^{\prime}\right)\right] .
\end{aligned}
$$

On the other hand, with the semiclassical approximation (i.e. $\theta_{1,2} \approx \theta_{1,2}^{\prime}$ ) taken into account, $S_{p}$ may be written as:

$$
S_{p} \approx-2 K \delta \theta_{2} \sin \left(\frac{\delta \theta_{1}}{2}\right) \cos \left[\frac{1}{2}\left(\frac{\theta_{1}+\theta_{1}^{\prime}}{2}-\frac{\theta_{2}+\theta_{2}^{\prime}}{2}\right)\right]-K \phi \cos \left[\frac{1}{2}\left(\frac{\theta_{1}+\theta_{1}^{\prime}}{2}-\frac{\theta_{2}+\theta_{2}^{\prime}}{2}\right)\right] .
$$

For sufficiently large $K,\left[\left(\theta_{1}+\theta_{1}^{\prime}\right)-\left(\theta_{2}+\theta_{2}^{\prime}\right)\right] / 4$ may be regarded as a random phase. Therefore, the exponent becomes self-averaging over this phase. Moreover, $\delta \theta_{1,2} \approx 0$. Finally, we employ the conventional hydrodynamic approximation, i.e. take into account the leading term in the $K \phi$-expansion only, to arrive at

$$
\begin{aligned}
& \exp \left(\frac{i S_{p}}{\hbar}\right) \rightarrow\left\langle\exp \left(\frac{i S_{p}}{\hbar}\right)\right\rangle \\
\approx & \left\langle\exp \left\{-\frac{i K \delta \theta_{1} \delta \theta_{2}}{\hbar} \cos \left[\frac{1}{2}\left(\frac{\theta_{1}+\theta_{1}^{\prime}}{2}-\frac{\theta_{2}+\theta_{2}^{\prime}}{2}\right)\right]\right\}\right\rangle \\
= & J_{0}\left(\frac{K \delta \theta_{1} \delta \theta_{2}}{\hbar}\right) .
\end{aligned}
$$

Let us insert Eqs. (45), (49), and (51), as well as Wigner transform of $\mathcal{A}, \mathcal{B}$ [cf. Eq. (14)], and $\mathcal{C}_{0}$ [cf. Eq. (24)] into Eq. (43), and integrate out $\left(l_{1}-l_{1}^{\prime}\right) / \hbar$, $\left(l_{4}-l_{4}^{\prime}\right) / \hbar,\left(l_{2}-l_{3}^{\prime}\right) / \hbar,\left(l_{3}-l_{2}^{\prime}\right) / \hbar,\left(l_{+}-l_{-}\right) / \hbar,\left(l_{+}^{\prime}-l_{-}^{\prime}\right) / \hbar$ and $\phi / \hbar$. As a result, we find that the semiclassical approximation for $\mathcal{I}_{q}$ is

$$
\begin{aligned}
\mathcal{I}_{q}\left(l, \theta ; l^{\prime}, \theta^{\prime}\right)= & \hat{\mathcal{V}}\left[\mathcal{A}_{\mathrm{W}}\left(l, \theta ; l^{\prime \prime}+\frac{\delta l_{2}}{2}, \theta^{\prime \prime}+\frac{\delta \theta_{1}}{2}\right)\right. \\
& \left.\times \mathcal{B}_{\mathrm{W}}\left(l^{\prime \prime}-\frac{\delta l_{2}}{2},-\theta^{\prime \prime}+\frac{\delta \theta_{1}}{2} ; l^{\prime}, \theta^{\prime}\right)\right],
\end{aligned}
$$

where the vertex operator $\hat{\mathcal{V}}$ is an integral operator: $\hat{\mathcal{V}} f\left(l, \theta ; l^{\prime}, \theta^{\prime} ; l^{\prime \prime}, \theta^{\prime \prime} ; \delta l_{2}, \delta \theta_{1}\right) \rightarrow(\hat{\mathcal{V}} f)\left(l, \theta ; l^{\prime}, \theta^{\prime}\right)$ (note that the variables: $l^{\prime \prime}, \theta^{\prime \prime}, \delta l_{2}$, and $\delta \theta_{1}$ in the function $f$ are integrated out) and is defined as

$$
\begin{aligned}
& (\hat{\mathcal{V}} f)\left(l, \theta ; l^{\prime}, \theta^{\prime}\right) \\
\equiv & \int \frac{d l^{\prime \prime} d \theta^{\prime \prime}}{2 \pi \hbar} \int \frac{d \delta l_{1} d \delta \theta_{1}}{2 \pi \hbar} \int \frac{d \delta l_{2} d \delta \theta_{2}}{2 \pi \hbar} \mathcal{X}\left(\delta l_{1}, \delta \theta_{1} ; \delta l_{2}, \delta \theta_{2}\right) \\
& \times \mathcal{C}_{0}\left(l^{\prime \prime}+\frac{\delta l_{1}}{2}, \theta^{\prime \prime}-\frac{\delta \theta_{2}}{2} ; l^{\prime \prime}-\frac{\delta l_{1}}{2},-\theta^{\prime \prime}-\frac{\delta \theta_{2}}{2}\right) \\
& \times f\left(l, \theta ; l^{\prime}, \theta^{\prime} ; l^{\prime \prime}, \theta^{\prime \prime} ; \delta l_{2}, \delta \theta_{1}\right)
\end{aligned}
$$

with

$$
\begin{aligned}
& \mathcal{X}\left(\delta l_{1}, \delta \theta_{1} ; \delta l_{2}, \delta \theta_{2}\right) \\
= & J_{0}\left(\frac{K \delta \theta_{1} \delta \theta_{2}}{\hbar}\right) \exp \left[\frac{i}{\hbar}\left(\delta l_{1} \delta \theta_{1}+\delta l_{2} \delta \theta_{2}\right)\right] .
\end{aligned}
$$

In Eq. (52) we introduced the following notations

$$
\begin{aligned}
l^{\prime \prime} & \equiv \frac{1}{2}\left(\frac{l_{2}+l_{3}^{\prime}}{2}+\frac{l_{3}+l_{2}^{\prime}}{2}\right) ; \\
\theta^{\prime \prime} & \equiv \frac{1}{2}\left(\frac{\theta_{1}+\theta_{1}^{\prime}}{2}-\frac{\theta_{2}+\theta_{2}^{\prime}}{2}\right),
\end{aligned}
$$

while the subscript $\mathrm{W}$ stands for Wigner transformation.

The minimal quantum wave packet posses the uncertainty: $\delta l_{1} \delta \theta_{1} \sim \hbar$ and $\delta l_{2} \delta \theta_{2} \sim \hbar$, as indicated in Eq. (54). Moreover, $\delta \theta_{1} \delta \theta_{2} \sim \hbar / K$. Such quantum wave packet determines the initial scale of the deviation of two nearby trajectories involved in the Cooperon [cf. Eq. (38)].

\section{Interaction vertex at the semiclassical level}

Applying the general expression Eq. (52) to Eq. (40), we find

$$
\begin{aligned}
& \delta \mathcal{D}\left(l, \theta ; l^{\prime}, \theta^{\prime}\right) \\
= & \hat{\mathcal{V}}\left[\mathcal{D}_{0}\left(l, \theta ; l^{\prime \prime}+\frac{\delta l_{2}}{2}, \theta^{\prime \prime}+\frac{\delta \theta_{1}}{2}\right)\left(\overleftarrow{P}_{T}-1\right)\right. \\
& \left.\times(\vec{P}-1) \mathcal{D}_{0}\left(l^{\prime \prime}-\frac{\delta l_{2}}{2},-\theta^{\prime \prime}+\frac{\delta \theta_{1}}{2} ; l^{\prime}, \theta^{\prime}\right)\right]
\end{aligned}
$$

Remarkably, this exact vertex $\delta \mathcal{D}$ is obtained without introducing any explicit regularization. In the next subsection we will show that it leads to the weak-localization correction to diffusion constant, which is similar to earlier findings for ballistic quantum dots $\underset{27}{27}$ In Appendix E we show that it is possible to introduce some artificial quantum disorder to QKR and the results obtained in the present work may be reproduced following the formalism of Ref. 27. 


\section{B. Weak localization correction to diffusion constant}

In this part, we show that the exact one-loop quantum correction, Eq. (56), may be cast into the conventional Hikami box structure with an additional, factor due to the Lyapunov region. As a result, the one-loop correction affects the diffusion equation through a frequencydependent renormalization of the diffusion coefficient: $D(\omega)=D_{c l}+\delta D(\omega)$. That is,

$$
\left[-i \omega-D(\omega) \nabla_{l}^{2}\right] \mathcal{D}_{0}=\hbar \delta\left(l-l^{\prime}\right) .
$$

We calculate then the quantum correction $\delta D(\omega)$ and find how it affects the angular momentum dispersion.

\section{Effects of the Lyapunov instability on the interaction vertex}

Since our aim is to describe the long-time phenomena, we expect that the typical scale of the angular momentum dispersion, say $L_{H}$, is large: $L_{H} \gg K$. Indeed the angular momentum is randomly spread in the interval $\in[-K, K]$ in a single kick. It is thus natural to expect much broader distribution after many kicks. This consideration justifies the expansion with respect to $K \nabla_{l}$ (hydrodynamic approximation). With such approximation, the exact vertex can be cast into the Hikami box.

With the help of the identity: $\vec{P} \mathcal{D}_{0}=\mathcal{D}_{0} \overleftarrow{P}_{T}$, which is proven in Appendix B. Eq. (56) may be rewritten as

$$
\begin{aligned}
& \delta \mathcal{D}\left(l, \theta ; l^{\prime}, \theta^{\prime}\right) \\
= & \hat{\mathcal{V}}\left\{\left[(\vec{P}-1) \mathcal{D}_{0}\left(l, \theta ; l^{\prime \prime}+\frac{\delta l_{2}}{2}, \theta^{\prime \prime}+\frac{\delta \theta_{1}}{2}\right)\right]\right. \\
& \left.\times\left[\mathcal{D}_{0}\left(l^{\prime \prime}-\frac{\delta l_{2}}{2},-\theta^{\prime \prime}+\frac{\delta \theta_{1}}{2} ; l^{\prime}, \theta^{\prime}\right)\left(\overleftarrow{P}_{T}-1\right)\right]\right\} .
\end{aligned}
$$

Since $\mathcal{X} \mathcal{C}_{0}$ has no dependence on $\left(l^{\prime \prime}, \theta^{\prime \prime}\right)$, Eq. (58) contains

$$
\begin{aligned}
I= & \int \frac{d l^{\prime \prime} d \theta^{\prime \prime}}{2 \pi k} \mathcal{D}_{0}\left(l, \theta ; l^{\prime \prime}+\frac{\delta l_{2}}{2}, \theta^{\prime \prime}+\frac{\delta \theta_{1}}{2}\right) \\
& \times \mathcal{D}_{0}\left(l^{\prime \prime}-\frac{\delta l_{2}}{2},-\theta^{\prime \prime}+\frac{\delta \theta_{1}}{2} ; l^{\prime}, \theta^{\prime}\right)
\end{aligned}
$$

To proceed further, we employ the following relation:

$$
\mathcal{D}_{0}\left(l, \theta ; l^{\prime}, \theta^{\prime}\right)=\mathcal{D}_{0}\left(l^{\prime},-\theta^{\prime} ; l,-\theta\right),
$$

which reflects the time-reversibility and its derivation is given in Appendix $\mathbb{B}$ The remaining procedure is fully analogous to calculation of the Cooperon developed in Sec. IIIC The only difference is in the boundary conditions. In fact, the angular deviation of two travelling nearby trajectories, $\delta \theta$ reaches a classical size $\left|\delta \theta^{\prime \prime}\right| \lesssim 1$ at some point in the phase space, say $\left(l_{n_{c}}, \theta_{n_{c}}\right)$. The later evolutions are independent. That is, the two Diffusons become self-averaging over the (random) paths connecting two remote ends, resulting in the factorization of the two averaged Diffusons as

$$
\begin{aligned}
& {\left[\mathcal{D}_{0}\left(l^{\prime \prime}+\frac{\delta l_{2}}{2},-\theta^{\prime \prime}-\frac{\delta \theta_{1}}{2} ; l,-\theta\right)\right.} \\
& \left.\times \mathcal{D}_{0}\left(l^{\prime \prime}-\frac{\delta l_{2}}{2},-\theta^{\prime \prime}+\frac{\delta \theta_{1}}{2} ; l^{\prime}, \theta^{\prime}\right)\right] \\
\rightarrow & \left\langle\mathcal{D}_{0}\left(l_{1}+\frac{\delta l^{\prime \prime}}{2},-\theta_{1}-\frac{\delta \theta^{\prime \prime}}{2} ; l,-\theta\right)\right\rangle \\
& \times\left\langle\mathcal{D}_{0}\left(l_{1}-\frac{\delta l^{\prime \prime}}{2},-\theta_{1}+\frac{\delta \theta^{\prime \prime}}{2} ; l^{\prime}, \theta^{\prime}\right)\right\rangle .
\end{aligned}
$$

Taking this boundary condition into account, we obtain:

$$
\begin{aligned}
I= & \mathcal{W}_{\mathrm{D}}(2 \omega) \int \frac{d l_{1} d \theta_{1}}{2 \pi \hbar}\left\langle\mathcal{D}_{0}\left(l, \theta ; l_{1}+\frac{\delta l^{\prime \prime}}{2}, \theta_{1}+\frac{\delta \theta^{\prime \prime}}{2}\right)\right\rangle \\
& \times\left\langle\mathcal{D}_{0}\left(l_{1}-\frac{\delta l^{\prime \prime}}{2},-\theta_{1}+\frac{\delta \theta^{\prime \prime}}{2} ; l^{\prime}, \theta^{\prime}\right)\right\rangle .
\end{aligned}
$$

Note that the two intermediate angular momenta deviate as $\delta l^{\prime \prime} / 2$. Such deviation is unimportant because the distribution with respect to the angular momentum fluctuates over the large scale $L_{H} \gg K \gg \delta l^{\prime \prime} / 2$. In Eq. (62) $\mathcal{W}_{\mathrm{D}}(\omega)$ is the same as $\mathcal{W}_{\mathrm{C}}(\omega)$ except that $t^{\mathrm{C}}$ is replaced by (with the logarithmic accuracy):

$$
t^{\mathrm{D}}=\frac{1}{\lambda}\left|\ln \frac{1}{\sqrt{\delta \theta^{2}+\delta l^{2} / K^{2}}}\right|
$$

where $\delta \theta$ is the initial angular separation of two nearby trajectories involved in the Diffuson side of the Lyapunov region. It is determined by the minimal quantum wave packet, i.e., by $\mathcal{X}$ [see Eq. (54)]. We then substitute Eqs. (53) and (62) into Eq. (58), and restore the operator under the average. As a result, we obtain:

$$
\begin{aligned}
& \delta \mathcal{D}\left(l, \theta ; l^{\prime}, \theta^{\prime}\right) \\
= & \mathcal{V} \int \frac{d l_{1} d \theta_{1}}{2 \pi \hbar}\left\{\left\langle\mathcal{D}_{0}\left(l, \theta ; l_{1}+\frac{\delta l^{\prime \prime}}{2}, \theta_{1}+\frac{\delta \theta^{\prime \prime}}{2}\right)\left(\overleftarrow{P}_{T}-1\right)\right\rangle\left\langle(\vec{P}-1) \mathcal{D}_{0}\left(l_{1}-\frac{\delta l^{\prime \prime}}{2},-\theta_{1}+\frac{\delta \theta^{\prime \prime}}{2} ; l^{\prime}, \theta^{\prime}\right)\right\rangle\right\}
\end{aligned}
$$


where

$$
\begin{aligned}
\mathcal{V} & \equiv \int \frac{d \delta l_{1} d \delta \theta_{1}}{2 \pi k} \int \frac{d \delta l_{2} d \delta \theta_{2}}{2 \pi k} \mathcal{W}_{\mathrm{D}}(2 \omega) \mathcal{X}\left(\delta l_{1}, \delta \theta_{1} ; \delta l_{2}, \delta \theta_{2}\right) \\
& \times \mathcal{C}_{0}\left(l^{\prime \prime}+\frac{\delta l_{1}}{2}, \theta^{\prime \prime}-\frac{\delta \theta_{2}}{2} ; l^{\prime \prime}-\frac{\delta l_{1}}{2},-\theta^{\prime \prime}-\frac{\delta \theta_{2}}{2}\right) .
\end{aligned}
$$

Here $\mathcal{V}$ may be regarded as the renormalized interaction strength. To further calculate it, we substitute Eq. (39) into Eq. (65). As a result, $\mathcal{V}$ is found to be

$$
\mathcal{V}=\Gamma(\omega) \int \frac{d \varphi}{2 \pi} \frac{\hbar}{-i \omega+D_{c l} \varphi^{2}}
$$

where

$$
\begin{aligned}
\Gamma(\omega) \equiv & \int \frac{d \delta l_{1} d \delta \theta_{1}}{2 \pi \hbar} \int \frac{d \delta l_{2} d \delta \theta_{2}}{2 \pi \hbar} \mathcal{W}_{\mathrm{C}}(2 \omega) \mathcal{W}_{\mathrm{D}}(2 \omega) \mathcal{X} \\
= & \int \frac{d \delta l_{1} d \delta \theta_{1}}{2 \pi \hbar} \int \frac{d \delta l_{2} d \delta \theta_{2}}{2 \pi \hbar} \\
& \times J_{0}\left(\frac{K \delta \theta_{1} \delta \theta_{2}}{\hbar}\right) \exp \left[\frac{i}{\hbar}\left(\delta l_{1} \delta \theta_{1}+\delta l_{2} \delta \theta_{2}\right)\right] \\
& \times \exp \left[\left(2 i \omega-\frac{2 \omega^{2} \lambda_{2}}{\lambda^{2}}\right)\left|z_{1}+z_{2}\right|\right]
\end{aligned}
$$

with

$$
z_{1}=\ln \sqrt{\delta \theta_{1}^{2}+\delta l_{2}^{2} / K^{2}}, \quad z_{2}=\ln \sqrt{\delta \theta_{2}^{2}+\delta l_{1}^{2} / K^{2}} .
$$

Rescaling $\delta \theta_{1,2}$ and $\delta l_{1,2}$ as

$$
\delta \theta_{1,2} \rightarrow \delta \theta_{1,2} / \sqrt{\hbar / K}, \quad \delta l_{1,2} \rightarrow \delta l_{1,2} / \sqrt{\hbar K}
$$

leads to

$$
\Gamma(\omega)=\exp \left(4 i \omega t_{E}-\frac{4 \omega^{2} \lambda_{2} t_{E}}{\lambda^{2}}\right) F(\omega),
$$

where $F(\omega)$ is

$$
\begin{aligned}
F(\omega)= & \int \frac{d l_{1} d \theta_{1}}{2 \pi} \int \frac{d l_{2} d \theta_{2}}{2 \pi} J_{0}\left(\theta_{1} \theta_{2}\right) \\
& \times \exp \left[i\left(l_{1} \theta_{1}+l_{2} \theta_{2}\right)\right] \\
& \times \exp \left[\left(2 i \omega-\frac{2 \omega^{2} \lambda_{2}}{\lambda^{2}}\right)\left|\widetilde{z}_{1}+\widetilde{z}_{2}\right|\right], \\
\widetilde{z}_{1}= & \ln \sqrt{\theta_{1}^{2}+l_{2}^{2}}, \quad \widetilde{z}_{2}=\ln \sqrt{\theta_{2}^{2}+l_{1}^{2}}
\end{aligned}
$$

Here $t_{E}=\left(t^{\mathrm{C}}+t^{\mathrm{D}}\right) / 2$, which is Eq. (2). Since $\widetilde{z}_{1,2} \sim 1$, in the limit $\omega, \omega \lambda_{2} / \lambda^{2} \ll 1$, the last exponent in Eq. (71) may be considered to be 1 . As a result, $F(\omega)=1$. Thus, we obtain:

$$
\Gamma(\omega)=\exp \left(4 i \omega t_{E}-\frac{4 \omega^{2} \lambda_{2} t_{E}}{\lambda^{2}}\right) .
$$

We point out that the position of minimal wave packetHikami box-can not be exactly located within the Lyapunov region. Indeed, this is reflected in the fact that the total duration within the Lyapunov region, i.e., $4 t_{E}$ actually does not depend on the exact boundary between Cooperon and Diffuson. Such feature originates from the chaotic nature of the classical motion in the phase space. At each full travel, the initial deviation, $\delta l_{1,2}$ $\left(\delta \theta_{1,2}\right)$ with respect to the reference trajectory expands in the backward (forward) time direction. Eventually $\delta l_{1} \delta \theta_{1}\left(\delta l_{2} \delta \theta_{2}\right)$ reaches some classical action $K$ (the typical scale of the classical action). Therefore, the total duration is

$$
4 t_{E}=\frac{1}{\lambda} \ln \frac{K}{\delta l_{1} \delta \theta_{1}}+\frac{1}{\lambda} \ln \frac{K}{\delta l_{2} \delta \theta_{2}} .
$$

Taking into account the uncertainty relation: $\delta l_{1} \delta \theta_{1} \approx$ $\delta l_{2} \delta \theta_{2} \approx k$, we find the total duration to be $4 t_{E}=$ $4 \lambda^{-1} \ln \sqrt{K / \hbar}$.

\section{Frequency-dependent diffusion coefficient}

The renormalized interaction vertex, Eq. 64) may be further cast into the conventional (diffusive) Hikami box upon further simplifications. As discussed above, the hydrodynamic expansion may be performed because of $K / L_{H} \ll 1$. This allows the further simplification of Eq. (64). One finds to the first order in the hydrodynamic expansion 


$$
\begin{aligned}
& \left\langle\vec{P} \mathcal{D}_{0}\left(l_{1}-\frac{\delta l^{\prime \prime}}{2},-\theta_{1}+\frac{\delta \theta^{\prime \prime}}{2} ; l^{\prime}, \theta^{\prime}\right)\right\rangle \\
= & \left\langle\mathcal{D}_{0}\left(l_{1}-\frac{\delta l^{\prime \prime}}{2}+K \sin \left[\theta_{1}-\frac{\delta \theta^{\prime \prime}}{2}+l_{1}-\frac{\delta l^{\prime \prime}}{2}\right],-\left[\theta_{1}-\frac{\delta \theta^{\prime \prime}}{2}+l_{1}-\frac{\delta l^{\prime \prime}}{2}\right] ; l^{\prime}, \theta^{\prime}\right)\right\rangle \\
\approx & \left\langle\left[1+K \sin \left(\theta_{1}-\frac{\delta \theta^{\prime \prime}}{2}+l_{1}-\frac{\delta l^{\prime \prime}}{2}\right) \nabla_{l_{1}}\right] \mathcal{D}_{0}\left(l_{1}-\frac{\delta l^{\prime \prime}}{2},-\left[\theta_{1}-\frac{\delta \theta^{\prime \prime}}{2}+l_{1}-\frac{\delta l^{\prime \prime}}{2}\right] ; l^{\prime}, \theta^{\prime}\right)\right\rangle \\
\approx & {\left[1+K \sin \left(\theta_{1}-\frac{\delta \theta^{\prime \prime}}{2}+l_{1}-\frac{\delta l^{\prime \prime}}{2}\right) \nabla_{l_{1}}\right]\left\langle\mathcal{D}_{0}\left(l_{1}-\frac{\delta l^{\prime \prime}}{2},-\left[\theta_{1}-\frac{\delta \theta^{\prime \prime}}{2}+l_{1}-\frac{\delta l^{\prime \prime}}{2}\right] ; l^{\prime}, \theta^{\prime}\right)\right\rangle . }
\end{aligned}
$$

The last line results from the fact that $\left\langle\mathcal{D}_{0}\right\rangle$ has weaker dependence on the angle compared to the sinusoidal term for sufficiently large $K$. Similarly,

$$
\begin{aligned}
& \left\langle\mathcal{D}_{0}\left(l, \theta ; l_{1}+\frac{\delta l^{\prime \prime}}{2}, \theta_{1}+\frac{\delta \theta^{\prime \prime}}{2}\right) \overleftarrow{P}_{T}\right\rangle \\
\approx & {\left[1+K \sin \left(\theta_{1}+\frac{\delta \theta^{\prime \prime}}{2}+l_{1}+\frac{\delta l^{\prime \prime}}{2}\right) \nabla_{l_{1}}\right]\left\langle\mathcal{D}_{0}\left(l, \theta ; l_{1}+\frac{\delta l^{\prime \prime}}{2}, \theta_{1}+\frac{\delta \theta^{\prime \prime}}{2}+l_{1}-\frac{\delta l^{\prime \prime}}{2}\right)\right\rangle }
\end{aligned}
$$

On the other hand, by shifting the overall angle factor,

$$
\begin{aligned}
& \int \frac{d l_{1} d \theta_{1}}{2 \pi \hbar}\left\langle\mathcal{D}_{0}\left(l_{1}-\frac{\delta l^{\prime \prime}}{2},-\left[\theta_{1}-\frac{\delta \theta^{\prime \prime}}{2}+l_{1}-\frac{\delta l^{\prime \prime}}{2}\right] ; l^{\prime}, \theta^{\prime}\right)\right\rangle\left\langle\mathcal{D}_{0}\left(l, \theta ; l_{1}+\frac{\delta l^{\prime \prime}}{2}, \theta_{1}+\frac{\delta \theta^{\prime \prime}}{2}+l_{1}-\frac{\delta l^{\prime \prime}}{2}\right)\right\rangle \\
= & \int \frac{d l_{1} d \theta_{1}}{2 \pi \hbar}\left\langle\mathcal{D}_{0}\left(l_{1}+\frac{\delta l^{\prime \prime}}{2},-\theta_{1}+\frac{\delta \theta^{\prime \prime}}{2} ; l^{\prime}, \theta^{\prime}\right)\right\rangle\left\langle\mathcal{D}_{0}\left(l, \theta ; l_{1}+\frac{\delta l^{\prime \prime}}{2}, \theta_{1}+\frac{\delta \theta^{\prime \prime}}{2}\right)\right\rangle .
\end{aligned}
$$

This arises from the uniform distribution with respect to the common angle in the product of the two averaged Diffusons.

We then substitute these two expansions: Eqs. (74) and (75), as well as Eq. (76) into Eq. (64). For sufficiently large $K$, the sinusoidal term is quasi-random and may be averaged over the angular region $[0,2 \pi]$. As a result, the linear term in the hydrodynamic expansion does not survive upon this averaging and the second order term must be kept. Finally we obtain:

$$
\begin{aligned}
\delta \mathcal{D}\left(l, \theta ; l^{\prime}, \theta^{\prime}\right) & =\mathcal{V} \int \frac{d l_{1} d \theta_{1}}{2 \pi \hbar} K^{2}\left\langle\sin \left(\theta_{1}-\frac{\delta \theta^{\prime \prime}}{2}\right) \sin \left(\theta_{1}+\frac{\delta \theta^{\prime \prime}}{2}\right)\right\rangle \nabla_{l_{1}^{\prime}} \nabla_{l_{1}^{\prime \prime}} \\
& \times\left.\left[\left\langle\mathcal{D}_{0}\left(l, \theta ; l_{1}^{\prime}+\frac{\delta l^{\prime \prime}}{2}, \theta_{1}+\frac{\delta \theta^{\prime \prime}}{2}\right)\right\rangle\left\langle\mathcal{D}_{0}\left(l_{1}^{\prime \prime}-\frac{\delta l^{\prime \prime}}{2},-\theta_{1}+\frac{\delta \theta^{\prime \prime}}{2} ; l^{\prime}, \theta^{\prime}\right)\right\rangle\right]\right|_{l_{1}^{\prime}=l_{1}^{\prime \prime}=l_{1}} .
\end{aligned}
$$

Since $\delta \theta^{\prime \prime} \ll 1 \sim \theta_{1}$,

$$
\begin{aligned}
& K^{2}\left\langle\sin \left(\theta_{1}-\frac{\delta \theta^{\prime \prime}}{2}\right) \sin \left(\theta_{1}+\frac{\delta \theta^{\prime \prime}}{2}\right)\right\rangle \\
\approx & K^{2}\left\langle\sin ^{2} \theta_{1}\right\rangle=2 D_{c l} .
\end{aligned}
$$

On the other hand, the Diffuson is smooth over the scale $\sim K$, hence we may also drop out $\delta l^{\prime \prime} / 2$ in Eq. (77). Finally $\delta \mathcal{D}$ is cast into

$$
\begin{array}{r}
\delta \mathcal{D}\left(l, \theta ; l^{\prime}, \theta^{\prime}\right)=2 \mathcal{V} \int \frac{d l_{1} d \theta_{1}}{2 \pi k} D_{c l} \nabla_{l_{1}^{\prime}} \nabla_{l_{1}^{\prime \prime}} \\
\times\left.\left[\left\langle\mathcal{D}_{0}\left(l, \theta ; l_{1}^{\prime}, \theta_{1}\right)\right\rangle\left\langle\mathcal{D}_{0}\left(l_{1}^{\prime \prime},-\theta_{1} ; l^{\prime}, \theta^{\prime}\right)\right\rangle\right]\right|_{l_{1}^{\prime}=l_{1}^{\prime \prime}=l_{1}} .
\end{array}
$$

The diffusion coefficient appearing in Eq. (79) is $D_{c l}=$ $K^{2} / 4$. We argue that if more kicks are reserved for the Hikami box, i.e., $(\hat{P}-1)^{n}, n>1$, then the diffusion coefficient will acquire the same higher order corrections as Eq. (23).

With the angle averaged out diffusion is retrieved. As a result, the one-loop correction, Eq. (79), is simplified as

$$
\begin{aligned}
\delta \mathcal{D}_{0}\left(l, l^{\prime}\right) & =\hbar^{-1} \int d l_{1} \mathcal{V} D_{c l}\left[\nabla_{l_{1}^{\prime}}^{2}+\nabla_{l_{1}^{\prime \prime}}^{2}\right] \\
& \times\left.\left[\mathcal{D}_{0}\left(l, l_{1}^{\prime}\right) \mathcal{D}_{0}\left(l_{1}^{\prime \prime}, l^{\prime}\right)\right]\right|_{l_{1}^{\prime}=l_{1}^{\prime \prime}=l_{1}} .
\end{aligned}
$$

Denote the Fourier transform of $\mathcal{D}_{0}\left(l, l^{\prime}\right)$, with respect to $l-l^{\prime}$ as $\mathcal{D}_{0}(\varphi ; \omega)$. Substituting Eq. (66) into Eq. (80) we find the Fourier transform one-loop correction:

$$
\delta \mathcal{D}_{0}(\varphi ; \omega)=\frac{k \Gamma(\omega) D_{c l} \varphi^{2}}{\left(-i \omega+D_{c l} \varphi^{2}\right)^{2}} \int \frac{d \phi}{\pi} \frac{1}{-i \omega+D_{c l} \phi^{2}},
$$


which leads to the one-loop quantum correction to the diffusion coefficient as

$$
\delta D(\omega)=-\frac{k D_{c l}}{\pi} \Gamma(\omega) \int \frac{d \phi}{-i \omega+D_{c l} \phi^{2}} .
$$

\section{Dispersion function}

One may express the time evolution of the angular momentum dispersion as: $\delta\left\langle l^{2}(t)\right\rangle \equiv\left\langle(l(t)-l(0))^{2}\right\rangle$ in terms of the frequency-dependent diffusion coefficient. In fact, by averaging over the angle, we may write $\delta\left\langle l^{2}(t)\right\rangle$ as

$$
\begin{aligned}
\delta\left\langle l^{2}(t)\right\rangle & =\sum_{l}\left(l-l^{\prime}\right)^{2}\left[\mathcal{D}_{0}\left(l, l^{\prime} ; t\right)-\mathcal{D}_{0}\left(l, l^{\prime} ; 0\right)\right] \\
& =-\left.\frac{\partial^{2}}{\partial \varphi^{2}}\left[\mathcal{D}_{0}(\varphi ; t)-\mathcal{D}_{0}(\varphi ; 0)\right]\right|_{\varphi \rightarrow 0}
\end{aligned}
$$

Substituting Eq. (57) into it, we obtain:

$$
\delta\left\langle l^{2}(t)\right\rangle=\int_{-\infty}^{\infty} \frac{d \omega}{\pi} \frac{1-e^{-i \omega t}}{\omega^{2}} D(\omega)
$$

For sufficiently large $K$ one may ignore the fluctuation of $\lambda$, i.e. put $\lambda_{2}=0$. Consequently, in the leading order in $k$ the momentum dispersion is found to be:

$$
\delta\left\langle l^{2}(t)\right\rangle=2 D_{c l} t-\frac{8 k \sqrt{D_{c l}}}{3 \sqrt{\pi}} \theta\left(t-4 t_{E}\right)\left(t-4 t_{E}\right)^{3 / 2},
$$

where $\theta(t)$ is the step function (long-dashed line on Fig. 2). The singularity at $t=4 t_{E}$ is rounded by the Ehrenfest time fluctuations-arising from finite $\lambda_{2}$ (full line on Fig. 2). Substituting Eqs. (72) and (82) into Eq. (84), we arrive at

$$
\begin{gathered}
\delta\left\langle l^{2}(t)\right\rangle-2 D_{c l} t=-\frac{4 k \sqrt{D_{c l}}}{3 \pi}\left(\delta t_{E}\right)^{3 / 2} \\
\times \int_{-\infty}^{\infty} d \tau \theta\left(\frac{t-4 t_{E}}{\delta t_{E}}-\tau\right)\left(\frac{t-4 t_{E}}{\delta t_{E}}-\tau\right)^{3 / 2} e^{-\frac{\tau^{2}}{16}}
\end{gathered}
$$

where $\delta t_{E}=\sqrt{\lambda_{2} t_{E} / \lambda^{2}}$. As a result,

$$
\delta\left\langle l^{2}(t)\right\rangle=2 D_{c l} t-\frac{\Gamma\left(\frac{5}{4}\right) k}{3 \pi / 64} \sqrt{D_{c l}}\left(\delta t_{E}\right)^{3 / 2} f\left(\frac{4 t_{E}-t}{\delta t_{E}}\right),
$$

where $f(0)=1$ and

$$
f(x)= \begin{cases}\frac{8 \sqrt{2} \Gamma\left(\frac{7}{2}\right)}{\Gamma\left(\frac{5}{4}\right)} x^{-5 / 2} e^{-x^{2} / 16} & \text { for } x \gg 1 \\ \frac{1}{8 \Gamma\left(\frac{5}{4}\right)}(-x)^{3 / 2} & \text { for }-x \gg 1\end{cases}
$$

This result completes the calculations of the one-loop weak-localization correction.

\section{WEAK DYNAMICAL LOCALIZATION OF QKR WITH BROKEN TIME-REVERSAL SYMMETRY}

To exploit farther similarities and differences of the dynamical localization of the QKR and the Anderson localization we discuss here effects of breaking the timereversal symmetry (TRS). In case of Anderson localization in a random potential the TRS may be broken, for example, by a static magnetic field. The latter provides different phases for clock-wise and anti-clock-wise propagating trajectories, destroying thus the systematic interference correction discussed in Section II. It does not ruin, though, the Anderson localization completely. Indeed, higher order corrections (the minimum possible is the two-loop one) may be interpreted as interference of trajectories travelling the loops in the same direction only (Diffuson only diagrams with no Cooperons). The static magnetic field does not affect such diagrams. As a result, the Anderson localization exists even in this case, albeit with somewhat larger localization length.

It has been thus of long interest to show that an analogous phenomena exists for the QKR as well23.34.39.40.41. Our additional motivation comes from consideration of the Lyapunov regime and its sensitivity to the TRS breaking. In particular, the one-loop (TRS invariant) correction was found to be delayed by $4 t_{E}$. Does this time interval remains to be protected against perturbative corrections in higher loop processes ? Is the delay time still the same? These questions are of particular interest if and when the leading one-loop correction is destroyed by TRS breaking.

To answer these questions we investigate the model, described by the following Hamiltonian

$$
\begin{aligned}
\hat{H}= & \frac{\hat{l}^{2}}{2}+K \sum_{n}[\cos \hat{\theta} \delta(t-2 n) \\
& +\cos (\hat{\theta}+\Phi) \delta(t-(2 n+1))] .
\end{aligned}
$$

Here the time-reversal symmetry is broken for generic $\Phi$ except for $\Phi=0, \pi$. First, we analyze suppression of the Cooperon (and thus the one-loop diagram) arising from $\Phi$. We then calculate the two-loop correction. In contrast to the one-loop correction, the two-loop one is robust against $\Phi$ because it contains (among others) a diagram without the Cooperons. If the TRS is broken, the long-time correction is given by the two-loop diagram depicted in Fig. 6 6 20,43,44. It differs from Fig. 1 in that the two interfering paths propagate together in the same direction, except inside the Hikami box, where they switch from one to the other. Due to such geometry, the two-loop correction is not sensitive to $\Phi$. It requires three successive travelling through the Lyapunov region (each taken $2 t_{E}$ time). We show thus that the weak localization correction, given by this diagram, is delayed by $6 t_{E}{ }^{44}$. 

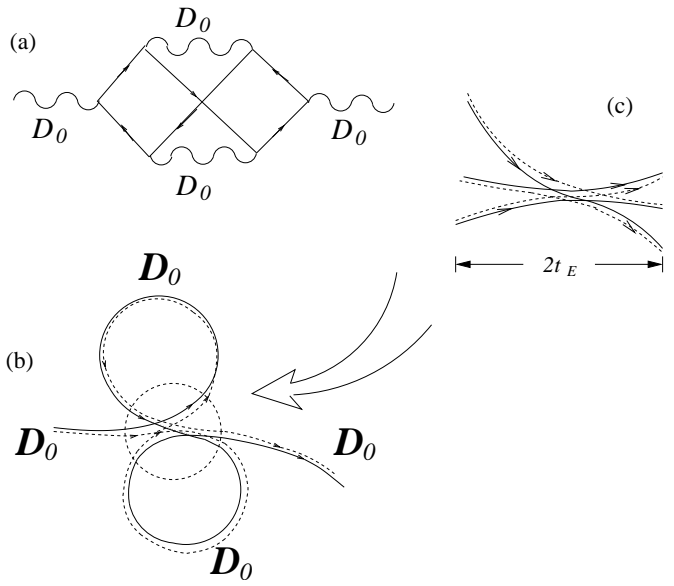

FIG. 6: The leading quantum correction to the densitydensity correlator in the absence of the time-reversal symmetry: (a) two-loop weak localization diagram; (b) its image in the momentum space; (c) Hikami box.

\section{A. Suppression of one-loop correction}

In the modified KR model, Eq. (89), the period is doubled and includes two kicking. The effective kicking operator appearing in the one-step evolution operator $U$ [cf. Eq. [13)] is replaced by

$$
\begin{aligned}
\hat{\mathcal{P}}_{J}^{\prime} \equiv & \exp \left[i \frac{K}{\hbar} \cos \hat{\theta}\right] \exp \left[\frac{i \hat{l}^{2}}{2 k}\right] \\
& \times \exp \left[i \frac{K}{\hbar} \cos (\hat{\theta}+\Phi)\right] .
\end{aligned}
$$

In the angular momentum representation, the matrix elements read as

$$
\begin{aligned}
\left\langle l_{+}\left|\hat{\mathcal{P}}_{J}^{\prime}\right| l_{+}^{\prime}\right\rangle= & \sum_{l_{1}} \int \frac{d \theta_{+}}{2 \pi} \int \frac{d \theta_{1}}{2 \pi} e^{-\frac{i}{\hbar} \theta_{+}\left(l_{+}-l_{1}\right)} e^{\frac{i K}{\hbar} \cos \theta_{+}} \\
& \times e^{\frac{i l_{1}^{2}}{2 \hbar}} e^{-\frac{i}{\hbar} \theta_{1}\left(l_{1}-l_{+}^{\prime}\right)} e^{\frac{i K}{\hbar} \cos \left(\theta_{1}+\Phi\right)}
\end{aligned}
$$

while its complex conjugation is

$$
\begin{aligned}
\left\langle l_{-}\left|\hat{\mathcal{P}}_{J}^{\prime}\right| l_{-}^{\prime}\right\rangle^{*}= & \sum_{l_{1}^{\prime}} \int \frac{d \theta_{-}}{2 \pi} \int \frac{d \theta_{1}^{\prime}}{2 \pi} e^{\frac{i}{k} \theta_{-}\left(l_{-}-l_{1}^{\prime}\right)} e^{-\frac{i K}{k} \cos \theta_{-}} \\
& \times e^{-\frac{i l_{1}^{\prime 2}}{2 k}} e^{\frac{i}{k} \theta_{1}^{\prime}\left(l_{1}^{\prime}-l_{-}^{\prime}\right)} e^{-\frac{i K}{k} \cos \left(\theta_{1}^{\prime}+\Phi\right)} \cdot(92)
\end{aligned}
$$

First we investigate the effects of $\Phi$ on the diffusive parts of Diffusons and Cooperons. To simplify the discussion we assume that $K$ is sufficiently large, implying that the memory about the angle is lost after a single kick. To find the self-energy of the Diffuson, we replace the kicking operator $J$ in Eq. (19) with $\hat{\mathcal{P}}_{J}^{\prime}$. Then we insert Eqs. (91) and (92) into it, putting $l_{1}=l_{1}^{\prime}$. Consequently, the diffusive pole is retrieved as $\theta_{+}-\theta_{-}=\theta_{1}-\theta_{1}^{\prime} \equiv k \varphi$, and the self-energy of the Diffuson is

$$
\begin{aligned}
& \left\langle l\left|\hat{\mathcal{P}}_{J}^{\prime}\right| l^{\prime \prime}\right\rangle\left\langle l\left|\hat{\mathcal{P}}_{J}^{\prime}\right| l^{\prime \prime}\right\rangle^{*} \\
= & \int \frac{d \varphi}{2 \pi} J_{0}^{2}\left(\frac{2 K}{\hbar} \sin \frac{k \varphi}{2}\right) e^{i \varphi\left(l-l^{\prime \prime}\right) .}
\end{aligned}
$$

This expression demonstrates the period doubling (Bessel function is squared) and implies that the Diffuson is not affected by $\Phi$.

For the diffusive Cooperon, the self-energy is $\left\langle l\left|\hat{\mathcal{P}}_{J}^{\prime}\right| l^{\prime \prime}\right\rangle\left\langle l^{\prime \prime}\left|\hat{\mathcal{P}}_{J}^{\prime}\right| l\right\rangle^{*}$. Inserting Eqs. (91) and (92) into it, and putting $l_{1}=l_{1}^{\prime}$, we find that the Cooperon has diffusive pole at

$$
\theta_{+}+\theta_{-}=\theta_{1}+\theta_{1}^{\prime} \equiv \hbar \varphi,
$$

and the self-energy is

$$
\begin{array}{r}
\left\langle l\left|\hat{\mathcal{P}}_{J}^{\prime}\right| l^{\prime \prime}\right\rangle\left\langle l^{\prime \prime}\left|\hat{\mathcal{P}}_{J}^{\prime}\right| l\right\rangle^{*}=\int \frac{d \varphi}{2 \pi} e^{i \varphi\left(l-l^{\prime \prime}\right)} \\
\times J_{0}\left(\frac{2 K}{\hbar} \sin \frac{\hbar \varphi}{2}\right) J_{0}\left[\frac{2 K}{\hbar} \sin \left(\frac{\hbar \varphi}{2}+\Phi\right)\right] .
\end{array}
$$

In the derivation above, we used the fact that for sufficiently large $K,\left(\theta_{+}-\theta_{-}\right) / 2$ and $\left(\theta_{1}-\theta_{1}^{\prime}\right) / 2$ is quasirandom and the self-averaging may be performed. With the Fourier transform with respect to time, the selfenergy leads to the diffusive Cooperon of the form:

$$
\begin{aligned}
& \left\langle\mathcal{C}_{0}\left(l, \theta ; l^{\prime},-\theta^{\prime} ; \omega\right)\right\rangle \\
= & \hbar \int \frac{d \varphi}{2 \pi} \frac{1}{-i \omega+\frac{1}{2} D_{c l}\left[\varphi^{2}+(\varphi+2 \Phi / \hbar)^{2}\right]}
\end{aligned}
$$

in the limit: $K \varphi, K \Phi / \hbar \ll 1$. Here $\left|l-l^{\prime}\right| \lesssim K, \mid \theta-$ $\theta^{\prime} \mid \sim 1$. According to Eq. (96) the relaxation time of the Cooperon is $\tau_{\Phi}=\left(D_{c l} \Phi^{2} / k^{2}\right)^{-1}$. At $\Phi \gtrsim k / \sqrt{D_{c l}} \sim$ $\hbar / K, \tau_{\Phi} \sim 1$ the Cooperon is completely suppressed.

The propagation in the Lyapunov region involves deterministic motion, which, is also affected by $\Phi$. Therefore the Lyapunov exponent $\lambda$, as well as its fluctuations $\lambda_{2}$ acquire some $\Phi$-dependence. However, since the propagator has no dependence on the center of mass, the functional form of $\mathcal{W}_{\mathrm{C}, \mathrm{D}}(2 \omega)$ remains unchanged. Thus, the procedure of Sec.IV B can be employed to show that the dispersion function is given by:

$\delta\left\langle l^{2}(t)\right\rangle=2 D_{c l} t-\frac{4 k \sqrt{D}_{c l}}{\sqrt{\pi}} \theta\left(t-4 t_{E}\right) \tau_{\Phi}^{3 / 2} h\left(\frac{t-4 t_{E}}{\tau_{\Phi}}\right)$,

where the function $h(x)$ is

$$
h(x)=\int_{0}^{x} d y_{1} \int_{0}^{\sqrt{y_{1}}} d y_{2} e^{-y_{2}^{2}} .
$$

Notice that here $t_{E}$ is a function of $\Phi$, i.e. $t_{E}(\Phi)=$ $\lambda(\Phi)^{-1} \ln \sqrt{K / \hbar}$. For simplicity, we neglected the fluctuations of $\lambda(\Phi)$, i.e. we put $\lambda_{2}=0$. In the region $t-4 t_{E} \gg \tau_{\Phi}$, the one-loop correction is exponentially suppressed. One is required, thus, to consider the higherloop corrections. The two-loop correction (Fig. 6) gives the leading weak dynamical localization correction. 


\section{B. Two-loop correction}

In principle the technique developed at the one-loop level can be employed to treat the two-loop case. However, this is technically quite involved and is not discussed here. To read out the frequency-dependent diffusion coefficient in an economical way, let us renormalize the standard results of the weak localization ${ }^{20}$ with an appropriate $t_{E}$-dependent factor [this procedure indeed is transparent at the one-loop level, Eq. [82)]. The renormalization factor for the two-loop geometries (see Fig. 6) were calculated in Ref. 44 in the context of the Lorentz gas. Adopting the analogy between the Lorentz gas model and the QKR, verified above on the one-loop level, one finds for the two-loop frequency-dependent correction to the diffusion coefficient

$$
\delta D(\omega)=-2 k^{2} D_{c l} \Gamma_{3}(\omega)\left[\int \frac{d \varphi}{2 \pi\left(-i \omega+D_{c l} \varphi^{2}\right)}\right]^{2},
$$

where 28.44

$$
\Gamma_{3}(\omega)=\exp \left(6 i \omega t_{E}-\frac{9 \omega^{2} \lambda_{2} t_{E}}{\lambda^{2}}\right) .
$$

As a result, the leading correction to the momentum dispersion in the case of broken TRS is given by:

$$
\delta\left\langle l^{2}(t)\right\rangle=2 D_{c l} t-\frac{1}{4} k^{2} \theta\left(t-6 t_{E}\right)\left(t-6 t_{E}\right)^{2} .
$$

Again we ignore $\lambda_{2}$ for simplicity. To develop the twoloop geometry, a minimal quantum wave packet must take time $t_{E}$ to expand into some macroscopic size, and vice versa. Within the logarithmic accuracy $t_{E}$ appearing here is the same as that in the one-loop correction. Therefore, the duration for a full travel through the Lyapunov region remains the same as the one-loop case, namely $2 t_{E}$ time. The two-loop geometry involves three successive visits. In the diagrammatical language, each leg of the 6-leg Hikami box (see Fig. 6 b) lasts time $t_{E}$. Thus, the weak localization correction, given by this diagram, is delayed by $6 t_{E} \underline{44}$.

\section{OBSERVATIONS OF CLASSICAL-TO-QUANTUM CROSSOVER IN REALISTIC DRIVEN SYSTEMS}

In this section, we discuss some possibilities for experimental observations of the predicted $t_{E}$-dependence of the classical-to-quantum crossover. The quantity to be measured is the dispersion function $\delta\left\langle l^{2}(t)\right\rangle$.

\section{A. Energy growth in cold atomic gases}

In the 90's unprecedented degree of control reached in experiments with ultra-cold atomic gases $\stackrel{45}{ }$ allowed to investigate various fundamental quantum phenomena. The advent of laser cooled atomic gases and standing wave optical pulses 2.3 .8 has opened the door to study quantum chaos experimentally. In an insightful paper, ${ }^{46}$ Graham, Schlautmann, and Zoller pointed out that atom optics may serve as a testing ground for quantum chaos. Shortly later, the idea came into realization with sodium atoms being cooled and trapped using the magneto-optical trap, subjected to a phase-modulated standing wave ${ }^{47}$. Later on, a realization of the QKR in atom optics was accomplished with the phase-modulated standing wave replaced by a pulsed standing wave ${ }^{2}$.

In an atom-optical experiment, typically $10^{6}$ sodium or cesium atoms are trapped and cooled down to $10 \mu \mathrm{K}$ using the conventional magneto-optical trap. After turning off the trapping fields, two linearly polarized, counter-propagated optical beams with the frequency $\omega_{L}$ are switched on, creating a spatially periodic potential: $V_{0} \cos \left(2 k_{L} x\right)$. Here $k_{L}=\omega_{L} / c$ is the laser wave number. Such optical lattice is controlled by the acousto-optical modulator as a pulse sequence with a profile $f(t)$. The pulse length $\tau_{p}$ may be much smaller than the period $T$. The atomic cloud, exposed to this pulsed optical lattice thereby, experiences a series of kicks. The evolution of the atomic momenta distribution is monitored after a certain number of kicks.

In experiments, the laser detuning $\Delta_{L} \equiv \omega_{L}-\omega_{0}$ from the resonance frequency $\omega_{0}$ is large compared to the excited-state decay rate. The dipole force due to Stark effect leads to the spatially-dependent shift of atomic levels. This results in an effective periodic potential imposed on the atomic cloud. One may model the center-of-mass motion of atoms with the single-particle time-dependent Hamiltonian as

$$
\hat{H}=\frac{\hat{p}^{2}}{2 m}+V_{0} \cos \left(2 k_{L} \hat{x}\right) \sum_{n=0}^{N} f(t-n T),
$$

where $m$ is the atomic mass, and $k_{L}$ is the laser wave number. The effective potential, $V_{0}$ is determined by the maximum Rabi frequency $\Omega$ as $V_{0}=\hbar \Omega^{2} / 8 \Delta_{L}$. The position $\hat{x}$, and the momentum $\hat{p}$ operators are canonically conjugated: $[\hat{x}, \hat{p}]=i \hbar$. The effective kicking strength $K$, and the Planck constant $k$ may be expressed then as:

$$
K=8 \omega_{r} T^{2} \tau_{p} V_{0} / \hbar, \quad \hbar=8 \omega_{r} T
$$

with the recoil frequency $\omega_{r}=\hbar k_{L}^{2} / 2 m$. With the rescaling: $x \rightarrow \theta \equiv 2 k_{L} x, p \rightarrow l \equiv\left(\hbar / 2 \hbar k_{L}\right) p, t \rightarrow t / T$, $f(t) \rightarrow f(t) / \tau_{p}, H \rightarrow(k T / \hbar) H$, one casts the Hamiltonian into Eq. (11) in the limit $\tau_{p} / T \rightarrow 0$ (with $K$ fixed) ${ }^{48}$.

So far many experimental efforts 2.8 have been focused on the parameter range where $k \gtrsim 2$, and thus $t_{E} \approx 1$. The dynamical localization has been observed as the saturation of the time-dependent momentum distribution width (i.e. energy absorption). To extract accurately the $t_{E}$-dependent crossover one needs a large separation between the relevant time scales: $1<t_{E}<t_{L}$. This requires to decrease $k$ down to $0.1-1$, which we 
hope to be soon within the reach for cold atomic gases experiments ${ }^{32.33}$.

\section{B. Charge fluctuations on Josephson grains}

The experimental realization of the QKR may be also feasible in experiments involving small nonequilibrium superconducting systems. One example is a small superconductive dot in contact with a bulk superconductor through two Josephson junctions $\frac{49}{}$. The bare Josephson coupling, $E_{J 0}$, is modulated via the external magnetic flux threading the SQUID loop 50.51.52: $E_{J}=$ $E_{J 0}\left|\cos \left(\pi B A_{\text {loop }} / \Phi_{0}\right)\right|$, where $A_{\text {loop }}$ is the area of the SQUID loop, and $\Phi_{0} \equiv h / 2 e$ is the superconducting flux quantum. If $B$ is modulated in a meander way, with the pulse length much smaller than the period $T$, the system may be modelled by the Hamiltonian:

$$
\hat{H}=\frac{\left(\hat{Q}-C V_{g}\right)^{2}}{2 C}-\bar{E}_{J} \cos \theta \sum_{n} T \delta(t-n T) .
$$

Here $\hat{\theta}$, and $\hat{Q}$ are the relative phase of the superconducting order parameter on the grain, and its charge, correspondingly. They are canonically conjugated: $[\hat{\theta}, \hat{Q}]=$ $2 e i$. In Eq. (104) $C$ is the capacitance, $V_{g}$ is the gate voltage, and $\bar{E}_{J}$ is the time-average Josephson coupling. Making change of the variables: $\hat{Q} \rightarrow \hat{l} \equiv \hbar \hat{Q} / 2 e$ and rescaling the relevant quantities as $t \rightarrow t / T, \hat{H} \rightarrow$ $8 E_{c} T^{2} \hat{H} / \hbar^{2}$, and $C V_{g} \rightarrow v_{g} \equiv \hbar C V_{g} / 2 e\left(E_{c}=e^{2} / 2 C\right)$, we cast the Hamiltonian above into QKR:

$$
\hat{H}=\frac{1}{2}\left(\hat{l}-v_{g}\right)^{2}-K \cos \theta \sum_{n} \delta(t-n) .
$$

Note that the sign difference in the kicking term is immaterial. Here the effective Planck's constant and kicking strength are

$$
\hbar=8 E_{c} T / \hbar, \quad K=8 E_{c} \bar{E}_{J} T^{2} / \hbar^{2},
$$

respectively.

The charge fluctuations are described by the charge dispersion: $\sim \delta\left\langle l^{2}(t)\right\rangle$. For sufficiently large $K$ and $t<4 t_{E}$ it is expected to increase linearly in time. At $t \gtrsim 4 t_{E}$, it should deviate from the linearity ${ }^{29}$. At longer time, $t \gg 4 t_{E}$ the $t^{3 / 2}$ power-law correction develops following the conventional weak localization theory ${ }^{1,18,19}$. This signals the onset of the localization phenomena. Eventually at $t \sim D_{c l} / \hbar^{2} \gg t_{E}$ the charge fluctuations saturate and do not grow any more upon further kicking.

\section{Charge fluctuations in superconducting nanocircuits}

Recent work ${ }^{34}$ suggested another kind of timemodulated small superconducting system. It is proposed

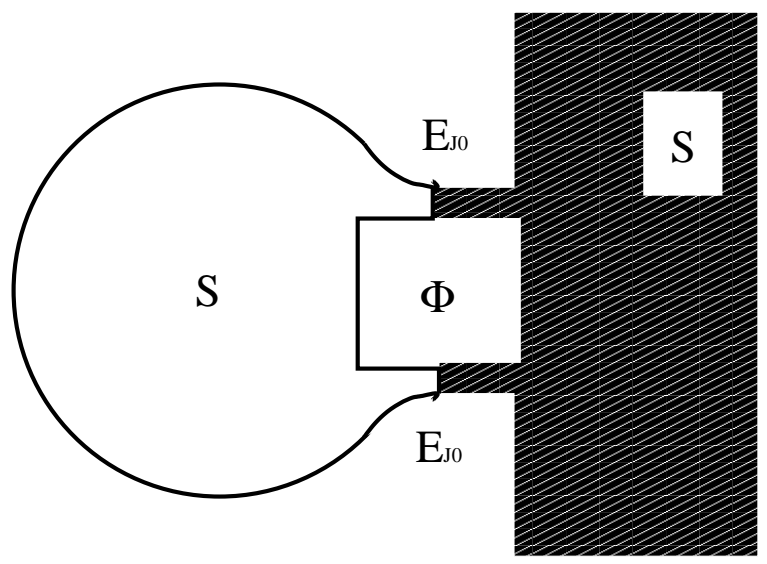

FIG. 7: The scheme of the driven Josephson grain: a superconducting dot (left) is coupled to a bulk superconductor (right) via a SQUID loop (middle). The flux $\Phi$ piercing the SQUID loop is time-dependent. It effectively modifies the bare Josephson coupling $E_{J 0}$ of the two junctions.

that a mechanically driven superconducting single electron transistor (SSET) may serve as a realization of the QKR. The system is based on a Cooper pair shuttle - a small superconducting island, periodically travelling between two macroscopic superconducting leads with the phases $\phi_{L}$ and $\phi_{R}$, respectively. Twice during each period, $T$, the shuttle meets one of the leads, experiencing a sudden Josephson coupling. The average coupling energy $\bar{E}_{J}$ is assumed to be much larger than the charging energy $E_{c}$. If the two leads are far enough from each other, the island never couples to both leads simultaneously. If the switching time is short, the time-dependent Josephson coupling may be mimicked by delta-pulses. Therefore, one may model the system with the following Hamiltonian:

$$
\begin{aligned}
\hat{H}= & -4 E_{c} \frac{\partial^{2}}{\partial \theta^{2}}-\bar{E}_{J} \sum_{n}[\cos \theta T \delta(t-2 n T) \\
& +\cos (\theta+\Phi) T \delta(t-(2 n+1) T)] .
\end{aligned}
$$

Here $\theta$ is the relative phase of the superconducting island with respect to the right lead. Remarkably, the phase difference across the two superconducting leads, $\Phi \equiv$ $\phi_{R}-\phi_{L}$ breaks the "time-reversal" symmetry, Eq. (8). The effective Planck constant and kicking strength remain the same as in Eq. (106). With the same rescaling as in Sec. VIB Eq. (107) is rewritten as Eq. (89).

The classical-to-quantum crossover is reflected in the nonequilibrium charge fluctuations of the superconducting island. In the case of $\Phi=0$, the situation is the same as Sec. VIB In the presence of a small phase bias across the two superconducting leads, i.e., $|\Phi| \lesssim \hbar / K$, the charge fluctuations are described by Eq. (97). For larger phase bias: $|\Phi| \gtrsim k / K$, the charge fluctuations are given by Eq. (101). 
(a)

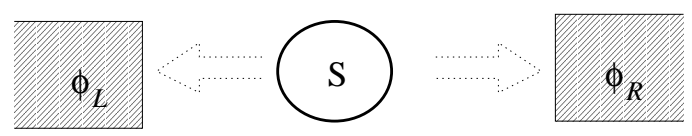

(b)

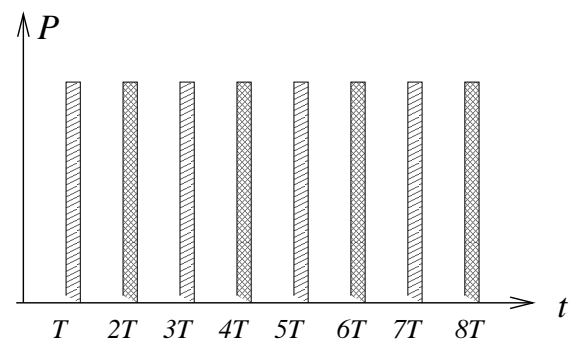

FIG. 8: A superconducting shuttle periodically travels between two superconducting leads with the phase $\phi_{L}$ and $\phi_{R}$, respectively (a). At every other period $2 T$, the shuttle experiences a sudden Josephson coupling with the left (right) lead.

\section{WEAK DYNAMICAL LOCALIZATION IN PRESENCE OF NOISE}

Due to the quantum interference nature of the weak dynamical localization, the effect may be strongly sensitive to noise. Indeed, noise effects are known to be of importance for the dynamical localization ${ }^{32.53 .54 .55}$. So far both experimental and theoretical studies have been primarily focused on $t_{E} \lesssim 1$. Below we shall consider how an external noise affects classical-to-quantum crossover at time scales $t \gtrsim t_{E}$. To this end, we will employ the technique of Sec. [IV] to investigate a noise-sensitivity of the weak dynamical localization Cooperon and Hikami box.

\section{A. Phase noises}

In proposed experiments involving small superconducting systems, the phase may fluctuate due to the influence of the dissipative measurement circuit. For the analytical treatment we focus on the particular kind of noises - the Gaussian phase noise. That is, the phase is assumed to fluctuate randomly in time: $\theta_{n} \rightarrow \theta_{n}+\zeta_{n}$. Here $\zeta_{n} \equiv \zeta_{t}$ is the noise that is assumed to be uncorrelated at different kicks $\left\langle\zeta_{n} \zeta_{n^{\prime}}\right\rangle=0$ for $n \neq n^{\prime}$. At a given kick the random phase $\zeta$ is supposed to be drawn from some periodic distribution function $P(\zeta)=(2 \pi)^{-1} \sum_{m} P_{m} e^{i m \zeta}$. For simplicity we shall assume that

$$
P_{m}=e^{-\sigma_{\varphi} m^{2}}
$$

where $\sigma_{\varphi}$ characterizes the strength of the noise.

\section{Strong phase noise}

The strong noise limit is characterized by $\sigma_{\varphi} \rightarrow \infty$, and thus $P(\zeta)=1 / 2 \pi$ being uniform distribution on $\zeta \in[0,2 \pi]$. We show first that the classical diffusion (without localization) is restored in this limit. The quantum density-density correlator satisfies (cf. Eq. (10)):

$$
\begin{aligned}
& \mathcal{D}_{\zeta}\left(l_{+}, l_{-} ; l_{+}^{\prime}, l_{-}^{\prime}\right)=e^{\frac{i\left(l_{+}^{2}-l_{-}^{2}\right)}{2 k}} \delta_{l_{+}, l_{+}^{\prime}} \delta_{l_{-}, l_{-}^{\prime}} \\
& +e^{i \omega} \sum_{l_{+}^{\prime \prime}, l_{-}^{\prime \prime}} \frac{(109}{\left\langle l_{+}|\hat{U}| l_{+}^{\prime \prime}\right\rangle\left\langle l_{-}|\hat{U}| l_{-}^{\prime \prime}\right\rangle^{*}} \mathcal{D}_{\zeta}\left(l_{+}^{\prime \prime}, l_{-}^{\prime \prime} ; l_{+}^{\prime}, l_{-}^{\prime} ; \omega\right),
\end{aligned}
$$

where the long bar stands for the average over the phase noise with respect to the uniform distribution, i.e.,

$$
\begin{aligned}
& \overline{\left\langle l_{+}|\hat{U}| l_{+}^{\prime \prime}\right\rangle\left\langle l_{-}|\hat{U}| l_{-}^{\prime \prime}\right\rangle^{*}} \\
\equiv & \int \frac{d \zeta}{2 \pi} \frac{d \theta_{+}}{2 \pi} \frac{d \theta_{-}}{2 \pi} \exp \left\{\frac{i K}{\hbar}\left[\cos \left(\theta_{+}+\zeta\right)-\cos \left(\theta_{-}+\zeta\right)\right]\right\} \\
& \times \exp \left[\frac{i\left(l_{+}^{2}-l_{-}^{2}\right)}{2 \hbar}+\frac{i \theta_{+}}{\hbar}\left(l_{+}-l_{+}^{\prime \prime}\right)-\frac{i \theta_{-}}{\hbar}\left(l_{-}-l_{-}^{\prime \prime}\right)\right] \\
= & \exp \left[\frac{i\left(l_{+}+l_{-}\right)\left(l_{+}-l_{-}\right)}{2 \hbar}\right] f\left(l_{+}-l_{+}^{\prime \prime}\right) \delta_{l_{+}-l_{-}, l_{+}^{\prime \prime}-l_{-}^{\prime \prime}} .
\end{aligned}
$$

In the last line $f(l)$ is defined as

$$
f(l) \equiv \int \frac{d \varphi}{2 \pi} J_{2 l}\left(\frac{2 K}{\hbar} \sin \frac{\hbar \varphi}{2}\right) .
$$

With the definition: $l \equiv\left(l_{+}+l_{-}\right) / 2, l^{\prime} \equiv\left(l_{+}^{\prime}+l_{-}^{\prime}\right) / 2$, and $\Delta l \equiv l_{+}-l_{-}$, the solution of Eq. (109) may be formally written as

$$
\begin{aligned}
& \mathcal{D}_{\zeta}\left(l, l^{\prime} ; \Delta l\right) \\
= & \exp \left[\frac{i l \Delta l}{2 k}\right] \delta_{l, l^{\prime}}+\sum_{n=1}^{\infty} e^{i \omega n} \sum_{l_{1}, \cdots, l_{n-1}} \exp \left[\frac{i \Delta l}{2 k} \sum_{k=1}^{n} l_{k}\right] \\
& \times f\left(l-l_{1}\right) f\left(l_{1}-l_{2}\right) \cdots f\left(l_{n-1}-l^{\prime}\right),
\end{aligned}
$$

where $l_{n} \equiv l^{\prime}$. As a result of the averaging over noises, $\mathcal{D}_{\zeta}$ has no $\zeta$-dependence. In the large $K$ limit, we expect the solution to be independent of $\left(l+l^{\prime}\right) / 2$. Thus, taking the average over $\left(l+l^{\prime}\right) / 2$, one finds:

$$
\begin{aligned}
\left\langle\mathcal{D}_{\zeta}\left(l, l^{\prime}\right)\right\rangle= & \delta_{l, l^{\prime}}+\sum_{n=1}^{\infty} e^{i \omega n} \sum_{l_{1}, \cdots, l_{n-1}} \\
& \times f\left(l-l_{1}\right) f\left(l_{1}-l_{2}\right) \cdots f\left(l_{n-1}-l^{\prime}\right) .
\end{aligned}
$$

Passing to Fourier representation, we find that it is nothing, but the classical diffuson, Eq. (21). It is worth mentioning that in the strong noise limit the diffusion constant is exactly $K^{2} / 4$. This is due to the fact that higher order corrections ${ }^{11}$ as well as quantum renormalization ${ }^{31.56}$ result from long-time correlation effects (cf. Appendix A). The latter is completely destroyed by the strong noise. 


\section{Weak phase noise limit}

We turn now to a more interesting case, relevant to the context discussed here, i.e., $\sigma_{\varphi} \ll 1$, where the noise only slightly suppresses the weak dynamical localization. For $\left\langle\zeta^{2}\right\rangle \sim \sigma_{\varphi} \ll 1, \cos (\varphi+\zeta) \approx \cos \varphi-\zeta \sin \varphi$.

Let us concentrate on the effect of the noise on the Cooperon. Upon every kicking, Cooperon acquires an additional phase: $K\left(\zeta_{t} \cos \theta_{+}-\zeta_{t^{\prime}} \cos \theta_{-}\right) / \hbar$. Recall that $t, t^{\prime}$ are counted from the two opposite ends of the loop trajectory, and $t+t^{\prime} \equiv T_{t}$ with $T_{t}$ being the total duration of the loop. Since $\zeta_{t}$ and $\zeta_{t^{\prime}}$ stand for noises at different moments, they are uncorrelated. Averaging the phase factor over them leads to the exponential suppression of of every step of the Cooperon ladder:

$$
\begin{aligned}
& \left\langle\exp \left(\frac{i K \zeta_{t}}{\hbar} \cos \theta_{+}\right) \exp \left(-\frac{i K \zeta_{t^{\prime}}}{\hbar} \cos \theta_{-}\right)\right\rangle_{\zeta_{t}, \zeta_{t^{\prime}}} \\
= & e^{-\frac{K^{2} \sigma_{\varphi}}{2 k^{2}}} I_{0}^{2}\left(\frac{K^{2} \sigma_{\varphi}}{4 \hbar^{2}}\right) .
\end{aligned}
$$

$\left(I_{0}(x)\right.$ is the modified Bessel function). This implies that the Cooperon is suppressed as

$$
\mathcal{C}_{0}(t) \rightarrow \mathcal{C}_{0}(t) e^{-t / t_{\varphi}}
$$

with the dephasing time defined as $t_{\varphi}=2 k^{2} /\left(K^{2} \sigma_{\varphi}\right)$. For $\sigma_{\varphi} \gtrsim(\hbar / K)^{2}, t_{\varphi} \sim 1$, the Cooperon mode is suppressed completely. It does not mean, however, that the classical diffusion is restored for such small $\sigma_{\varphi}$. Indeed, the higher order loop corrections, that include the Diffusons only (cf. Fig. 6) may still survive such level of the noise and lead to the dynamical localization. To verify if this is the case, one needs to study the effect of the week noise on Hikami box.

Starting from Eqs. (48) and (55), we observe that $\delta \theta_{1}$ and $\theta^{\prime \prime}$ are independently shifted by the noise, i.e., $\delta \theta_{1} \rightarrow$ $\delta \theta_{1}^{\prime} \equiv \delta \theta_{1}+\zeta_{1}, \theta^{\prime \prime} \rightarrow \theta^{\prime \prime}+\zeta_{2}$. As a result, instead of Eq. (154), $\mathcal{X}$ is given by

$$
\begin{aligned}
& \mathcal{X}\left(\delta l_{1}, \delta \theta_{1} ; \delta l_{2}, \delta \theta_{2}\right) \\
\rightarrow & \exp \left(\frac{i K \zeta \delta \theta_{1}^{\prime} \delta \theta_{2}}{\hbar} \sin \theta^{\prime \prime}\right) \mathcal{X}\left(\delta l_{1}, \delta \theta_{1}^{\prime} ; \delta l_{2}, \delta \theta_{2}\right) .
\end{aligned}
$$

Upon averaging over $\zeta$, Eq. (116) leads to the the exponential suppression of the minimal quantum wave packet (Hikami box) as

$$
\exp \left[-\left(\frac{K \delta \theta_{1}^{\prime} \delta \theta_{2}}{2 \hbar}\right)^{2} \sigma_{\varphi}\right] \mathcal{X}\left(\delta l_{1}, \delta \theta_{1}^{\prime} ; \delta l_{2}, \delta \theta_{2}\right) .
$$

From here we see that the weak phase noises $\left(\sigma_{\varphi} \ll\right.$ 1) does not substantially affect Hikami box. Indeed, the interaction vertex is significantly suppressed only at $\sigma_{\varphi} \sim 1$. This means that the effect of phase noises on Hikami box may be ignored compared to the dephasing of Cooperon.
As a result, the intermediate intensity noise $(k / K)^{2} \ll$ $\sigma_{\varphi} \ll 1$ acts, to much extent, as a TRS breaking perturbation. It suppresses the Cooperon corrections, leaving the Diffuson ones (the simplest being the two loop one Fig. 6] intact.

Above we find that the Diffuson-Cooperon couplingminimal wave packet, is suppressed by large enough phase noises. This picture is naturally expected to be applicable for higher order interaction vertex also. The latter is essentially responsible for the onset of the weak dynamical localization in Diffuson-only diagrams. This picture may be considered to be the precursor of the restoration of diffusion for larger noises.

\section{B. Amplitude noise}

In cold atoms experiments, the optical pulse power may fluctuate with time and thus the series is not perfectly periodic. This then leads to the noise in the kicking amplitude, i.e. the stochastic parameter $K$ is replaced by $K+\eta_{n}$, where $\eta_{n}=\eta_{t}$ is a random amplitude fluctuation. The effects on dynamical localization of such kind of noise have been under intensified experimental investigations $32,54.55$. A central issue addressed is, whether the dynamical localization is destroyed completely by the noise. We concentrate here on a weak noise limit.

To simplify analytical estimations below let us assume that $\eta_{n}$ is the white-noise, Gaussian noise, namely $\left\langle\eta_{n} \eta_{n^{\prime}}\right\rangle=\sigma_{K} \delta_{n n^{\prime}}$. Here $\sigma_{\mathrm{K}}$ characterizes the strength of the noise. Below we consider the limiting case of $\sigma_{\mathrm{K}} \ll 1$, where the amplitude noise only slightly suppresses the weak dynamical localization.

For the Diffuson the one-step quantum propagator acquires an additional phase as $\eta_{t}\left(\cos \theta_{+}-\cos \theta_{-}\right)$. Here $\theta_{+}, \theta_{-}$stand for the phases of retarded/advanced Green's functions at the kicks. Passing to the semiclassical limit, i.e., $\left|\theta_{+}-\theta_{-}\right| \ll 1$ and averaging over the noise, we find that the Diffuson is affected via renormalization of $D_{c l}$ by a small correction $\delta D_{c l} \sim \sigma_{\mathrm{K}} \ll D_{c l}$ (A detailed study in this direction was presented in Ref. 33.). This correction is not responsible for the destruction of the dynamical localization. It is the effect of the noise on the Cooperon and Hikami box that eventually may lead to the restoration of the classical diffusion.

In the case of the amplitude noise the effect on the Cooperon is fully analogous to the case of the phase noise, i.e. dephasing. Upon every kicking, the Cooperon acquires an additional phase: $\left(\eta_{t} \cos \theta_{+}-\eta_{t^{\prime}} \cos \theta_{-}\right) / \hbar$ with $t, t^{\prime}$ counted from the opposite ends respectively, such that $t+t^{\prime} \equiv T_{t}$ with $T_{t}$ being the total duration of the loop. Upon averaging over the independent noises $\eta_{t}$ and $\eta_{t^{\prime}}$, this phase factor leads to the exponential sup- 
pression of a single step of the Cooperon as:

$$
\begin{aligned}
& \left\langle\exp \left(\frac{i \eta_{t}}{\hbar} \cos \theta_{+}\right) \exp \left(-\frac{i \eta_{t^{\prime}}}{\hbar} \cos \theta_{-}\right)\right\rangle_{\eta_{t}, \eta_{t^{\prime}}} \\
= & \exp \left[-\frac{\sigma_{\mathrm{K}}}{2 k^{2}}\right] I_{0}^{2}\left(\frac{\sigma_{\mathrm{K}}}{4 k^{2}}\right) .
\end{aligned}
$$

This implies that the Cooperon is dephased as Eq. (115) with the dephasing time being $\tau_{\mathrm{K}}=2 k^{2} / \sigma_{\mathrm{K}}$. For $\sigma_{\mathrm{K}} \gtrsim k^{2}, \tau_{\mathrm{K}} \sim 1$, the Cooperon mode is suppressed completely thus only higher order terms (Diffusons only) may be responsible for the dynamical localization. It remains open to estimate the noise amplitude that destroys Diffuson-only weak dynamical localization.

\section{Effects of finite dephasing time}

We saw above that every realization of the QKR may involve various noises in realistic experimental environments. As a result, there exist various dephasing mechanisms. The effective dephasing rate is the sum over all dephasing rates, namely

$$
\frac{1}{\tau_{\varphi}}=\sum_{k} \frac{1}{\tau_{k}}
$$

To simplify qualitative discussions, in this part we focus on effects arising from weak dephsing such that $4 t_{E} \ll \tau_{\varphi}$. First we show that the effective Ehrenfest time is shortened. In fact, the additional weak noises enhance the rate of angular deviation spread according to:

$$
\frac{d}{d t} \delta \theta^{2}(t)=2 \lambda \delta \theta^{2}(t)+\frac{1}{\tau_{\varphi}} .
$$

Recall that the first term on the right hand side results from the Lyapunov instability. The solution is easily found, i.e., $\delta \theta^{2}(t)=\left[\delta \theta_{1,2}+\left(2 \lambda \tau_{\varphi}\right)^{-1}\right] e^{\lambda t}-\left(2 \lambda \tau_{\varphi}\right)^{-1}$. Here $\delta \theta_{1,2}$ are the initial angular deviation of Diffuson/Cooperon, respectively. At $t^{\mathrm{D} *}$ (or $t^{\mathrm{C} *}$ ), $\delta \theta \sim 1$. The solution then gives

$$
t^{\mathrm{D}, \mathrm{C} *} \approx \frac{1}{\lambda}\left|\ln \frac{1}{\sqrt{\delta \theta_{1,2}^{2}+\left(2 \lambda \tau_{\varphi}\right)^{-1}}}\right| .
$$

Thus, the effective duration of a full travel through the Lyapunov region is found to be

$$
\begin{aligned}
& t^{\mathrm{D} *}+t^{\mathrm{C} *}= \\
& \left|\ln \frac{1}{\sqrt{\left(\delta \theta_{1} \delta \theta_{2}\right)^{2}+\left(\delta \theta_{1}^{2}+\delta \theta_{2}^{2}\right)\left(2 \lambda \tau_{\varphi}\right)^{-1}+\left(2 \lambda \tau_{\varphi}\right)^{-2}}}\right| \\
\leq & \frac{1}{\lambda}\left|\ln \frac{1}{\left|\delta \theta_{1} \delta \theta_{2}\right|+\left(2 \lambda \tau_{\varphi}\right)^{-1}}\right| \\
\sim & \frac{2}{\lambda}\left|\ln \frac{1}{\sqrt{\frac{\hbar}{K}}+\left(2 \lambda \tau_{\varphi}\right)^{-1}}\right|=2 t_{E}^{*} .
\end{aligned}
$$

The effective Ehrenfest time $t_{E}^{*}<t_{E}$. With $t_{E}^{*}$ substituted into the renormalization factors $\mathcal{W}_{\mathrm{C}}$ and $\mathcal{W}_{\mathrm{D}}$, and taking into account the dephasing of diffusive Cooperon, the one-loop correction, Eq. (82), is modified as

$$
\begin{aligned}
\delta D(\omega)= & -\frac{k D_{c l}}{\pi} \mathcal{W}_{\mathrm{D}}(2 \omega) \mathcal{W}_{\mathrm{C}}(2 \omega) \\
& \times \int \frac{d \varphi}{-i \omega+D_{c l} \varphi^{2}+\frac{1}{\tau_{\varphi}}} .
\end{aligned}
$$

Taking the Fourier transform with respect to $\omega$, one find the momentum dispersion to be

$$
\begin{aligned}
& \delta\left\langle l^{2}(t)\right\rangle \\
= & 2 D_{c l} t-\frac{8 k \sqrt{D_{c l}}}{3 \sqrt{\pi}} \theta\left(t-4 t_{E}^{*}\right)\left(t-4 t_{E}^{*}\right)^{3 / 2} e^{-\frac{t-4 t_{E}^{*}}{\tau_{\varphi}}} .
\end{aligned}
$$

Again $\lambda_{2}=0$. According to Eq. (124) the quantum correction is suppressed at $t \gtrsim 4 t_{E}^{*}+\tau_{\varphi}$.

One should keep in mind that in realistic experiments, like atom-optical ones the Hamiltonian of QKR, Eq. (11) may be an oversimplification. Therefore, some restriction on the validity of the present result will be imposed. For example, the cold atoms experiment involves collisions between the atoms, which lead to another dephasing mechanism. Let us estimate the corresponding collision dephasing time, $\tau_{s}$. The two-particle scattering mean free path is known to be $l_{s} \approx 1 /\left(n a^{2}\right)$, where $n$ is the atomic concentration and $a$ is the $s$-wave scattering length. The corresponding dimensionless scattering time is $\tau_{s}=l_{s} /(T v)$, where $v$ is a typical atomic velocity that may be estimated as $v \approx \hbar k_{L}|l| / \hbar m \approx \hbar k_{L} \sqrt{D_{c l} \tau_{s}} / k m$. This leads to the self-consistent estimate of the dimensionless dephasing time:

$$
\tau_{s} \approx\left(l_{s} k_{L} / K\right)^{2 / 3} .
$$

Once again to observe the classical-to-quantum crossover the inequality $4 t_{E} \lesssim \tau_{s}$ should be valid.

\section{CONCLUSIONS}

In this paper, we developed an analytical theory to incorporate systematically the Ehrenfest time, $t_{E}$, into the weak dynamical localization. We map the loop expansion, central to the theory of weak dynamical localization onto the interference of paths in the configuration space with a certain loop geometry. To propagate along such paths a time longer than $2 m t_{E}(m=2,3, \cdots)$ (where $m$ is determined by a specific loop geometry) is needed. We establish thus that the onset of the dynamical localization is delayed by the multiples of $2 t_{E}$. In particular, for QKR, the delay is $4 t_{E}$, while for systems with broken time-reversal symmetry it is $6 t_{E}$. At shorter times, quantum corrections to the linear dispersion (classical diffusion) do exist. However, they results only in the renormalization of the frequency-independent diffusion coefficient, and thus are not responsible for the onset of the dynamical localization. 
Our quantitative predictions are based on the loop expansion and are essentially perturbative. They are closely analogous to the weak Anderson localization in ballistic systems. They may be considered as a further support for the long-standing conjecture of Ref. 13 about the similarity between the dynamical localization and Anderson localization for irrational $k / 4 \pi 23$. The perturbative corrections are responsible only for the early evolution of the dispersion function, i.e., the suppression of the classical diffusion. At longer time $t \gtrsim t_{L}$, the strong dynamical localization is expected to develop, as supported by arguments based on supersymmetric diffusive $\sigma$ mode ${ }^{19}$. However, it still remains a challenge to prove this conjecture in the presence of finite $t_{E}$.

Although our analytical treatment is developed for the QKR, we believe that it may be extended to more general quantum driven systems. The essential requirements imposed on the dynamics of an underlying classical system are: (i) area-preserving; and (ii) the existence of classical stochastic diffusion (subject to certain symmetry). Provided (i) and (ii) are satisfied, we expect the functional form of the weak localization corrections to be the same as derived above. The specific of chaotic motion enters through modifying the classical quantities such as diffusion coefficient, Lyapunov exponent and its fluctuations - such problem is purely classical and, in general, may be resolved (say numerically).

Technically, the most important part of this work is the derivation of the one-loop vertex (Hikami box) without introduction of a regularization. This allows to analyze accurately the minimal quantum wave packet. As a result, the Ehrenfest time is quantitatively defined (with the logarithmic accuracy) as the time needed to expand an initial minimal wave packet up to a macroscopic size $\mathrm{e}^{21,22}$. Alternatively, the minimal wave packet may be analyzed within the Moyal formalism 37.57 . A study along this line has been reported recently in the context of ballistic supersymmetric $\sigma$-mode ${ }^{30}$. This supports the conjecture of Ref. 27 that the Ehrenfest time should not depend on the regularization, since the later is only intended to mimic the effect of quantum diffraction. Indeed, in accord with Ref. 27, our current results may be fully reproduced by introducing a proper regularization ${ }^{37}$ to the supersymmetric $\sigma$ model developed for QKR ${ }^{19}$.

The quantitative predictions made for the $t_{E^{-}}$ dependent classical-to-quantum crossover in QKR may be suitable for experimental verifications in various contexts. In particular, already existing experiments on the dynamical localization in the energy growth of ultracold atomic gases have greatly contributed to understanding of this crossover. Our quantitative predictions are expected to be accurate in the asymptotic regime $\hbar<1<K$. For their quantitative verification, it is thus highly desirable to decrease $k$ down to $0.1-1$. We also pointed out that some periodically driven mesoscopic superconducting structures may be suitable for realizations of the QKR. The dynamical localization and classicalto-quantum crossover in these systems are observable by monitoring charge fluctuations of the superconducting island. It is important to mention that, all realistic experiments introduce noise and thus a finite dephasing time $\tau_{\varphi}$. We have shown here that for an observation of the $t_{E}$-dependent crossover, the condition $4 t_{E} \lesssim \tau_{\varphi}$ must be satisfied.

\section{Acknowledgments}

We have greatly benefited from discussions with A. Altland, D. Basko, S. Fishman, J. Liu and C. Zhang. We thank L. Glazman for pointing Ref. 35 to us. We are grateful to Abdus Salam International Center for Theoretical Physics, where part of this work was done, for its hospitality. C. T. and A. L. are supported by NSF Grant No. DMR-0120702 and DMR-0439026. A. K. is A. P. Sloan fellow and supported by the NSF grant No. DMR-0405212.

\section{APPENDIX A: FINITE TIME CORRELATION EFFECTS ON THE SELF-ENERGY}

In this Appendix we study the higher order time correlation effects, starting from the exact quantum densitydensity correlator, Eq. (12). In particular, we clarify that in the semiclassical limit, the higher order corrections, namely Eq. (23), (see Ref. 11) to the diffusion constant, i.e., $K^{2} / 4$ are found.

For an arbitrary $\tau_{c}>1 \mathrm{Eq}$. (18) is replaced by:

$$
\mathcal{D}_{0}\left(l, l^{\prime}\right)=\delta_{l, l^{\prime}}+\sum_{l^{\prime \prime}} \Sigma\left(l, l^{\prime \prime}\right) \mathcal{D}_{0}\left(l^{\prime \prime}, l^{\prime}\right)
$$

Here the self-energy, $\Sigma\left(l, l^{\prime \prime}\right)$ is given by

$$
\begin{aligned}
& \Sigma\left(l, l^{\prime \prime}\right)=\sum_{n=1}^{\tau_{c}} e^{i \omega n}\left\langle l\left|\hat{U}^{n}\right| l^{\prime \prime}\right\rangle\left\langle l\left|\hat{U}^{n}\right| l^{\prime \prime}\right\rangle^{*} \\
= & e^{i \omega} U_{l, l^{\prime \prime}} U_{l, l^{\prime \prime}}^{*}+\sum_{n=2}^{\tau_{c}} e^{i \omega n} \sum_{l_{1+}, l_{1-}} \ldots \sum_{l_{(n-1)+}, l_{(n-1)-}} \\
& \times \prod_{k=1}^{n-1} U_{l_{k+}, l_{(k+1)+}} U_{l_{k-}, l_{(k+1)-}}
\end{aligned}
$$

Note that $l_{0}=l_{0}^{\prime} \equiv l, l_{n}=l_{n}^{\prime} \equiv l^{\prime \prime}$, and $l_{k} \neq l_{k}^{\prime}$ for $0<k<n$. Here in order to simplify the notation we denote the matrix elements $\left\langle l_{k+}|\hat{U}| l_{(k+1)+}\right\rangle$ as $U_{l_{k+}, l_{(k+1)+}}$, and similarly for their complex conjugates. These matrix 
elements may be written explicitly as

$$
\begin{aligned}
U_{l_{k+}, l_{(k+1)+}}=\int \frac{d \theta_{(k+1)+}}{2 \pi} \exp \left[\frac{i l_{(k+1)+}^{2}}{2 k}\right. \\
\left.+\frac{i K}{\hbar} \cos \theta_{(k+1)+}-\frac{i}{\hbar}\left(l_{k+}-l_{(k+1)+}\right) \theta_{(k+1)+}\right], \\
U_{l_{k-,}^{*} l_{(k+1)-}}=\int \frac{d \theta_{(k+1)-}}{2 \pi} \exp \left[-\frac{i l_{(k+1)-}^{2}}{2 k}\right. \\
\left.-\frac{i K}{\hbar} \cos \theta_{(k+1)-}+\frac{i}{\hbar}\left(l_{k-}-l_{(k+1)-}\right) \theta_{(k+1)-}\right] .
\end{aligned}
$$

To proceed further, we introduce the following quantities:

$$
m_{k}=\left(l_{k+}-l_{k-}\right) / \hbar, \quad q_{k}=\left(\theta_{k+}-\theta_{k-}\right) / \hbar .
$$

Then with the substitution of Eq. A3 we rewrite $U_{l_{k+}, l_{(k+1)+}} U_{l_{k-}, l_{(k+1)-}}$ as

$$
\begin{aligned}
& U_{l_{k+}, l_{(k+1)+}} U_{l_{k-}, l_{(k+1)-}}^{*} \\
= & \iint \frac{d \theta_{(k+1)+}+\frac{d \theta_{(k+1)-}}{2 \pi} \exp \left\{i m_{k+1} \frac{l_{(k+1)+}+l_{(k+1)-}}{2 \pi}\right.}{2} \\
& -\frac{2 i K}{k} \sin \frac{k q_{k+1}}{2} \sin \frac{\theta_{(k+1)+}+\theta_{(k+1)-}}{2} \\
& -i q_{k+1}\left[\frac{l_{k+}+l_{k-}}{2}-\frac{l_{(k+1)+}+l_{(k+1)-}}{2}\right] \\
& \left.+i\left(m_{k}-m_{k+1}\right) \frac{\theta_{(k+1)+}+\theta_{(k+1)-}}{2}\right\} .
\end{aligned}
$$

Furthermore, we insert the Fourier transform:

$$
\begin{aligned}
& \exp \left[\frac{2 i K}{\hbar} \sin \theta \sin \frac{k q}{2}\right] \\
= & \sum_{n} J_{n \operatorname{sgn} q}\left(\frac{2 K}{\hbar} \sin \frac{k q}{2}\right) e^{i n \theta}
\end{aligned}
$$

into it with sgn denoting the sign of $q$. Then Eq. A5 is substituted into Eq. A2 . With the sum with respect to $\left(l_{k+}+l_{k-}\right) / 2(k=0,1,2, \cdots)$ and the integral with respect to $\left(\theta_{k+}+\theta_{k-}\right) / 2$ performed, eventually Eq. (A2) is reduced to

$$
\begin{aligned}
& \Sigma\left(l, l^{\prime \prime}\right)=e^{i \omega} J_{0}\left(\frac{2 K}{\hbar} \sin k q_{0}\right) \\
& +\sum_{r=2}^{\tau_{c}} e^{i \omega r} \sum_{n_{1}, n_{2}, \cdots, n_{r}} \sum_{m_{1}, m_{2}, \cdots, m_{r-1}} \sum_{q_{0}, q_{1}, \cdots, q_{r-1}} \prod_{k=1}^{r} \\
& \times J_{n_{k} \operatorname{sgn} q_{k-1}}\left(\frac{2 K}{\hbar}\left|\sin \frac{k q_{k-1}}{2}\right|\right) \\
& \times \delta_{q_{k}-q_{k-1},-m_{k}} \delta_{m_{k}-m_{k-1},-n_{k} \operatorname{sgn} q_{k-1}} .
\end{aligned}
$$

Shortly later we will see that the following relations, implied by the two Kroneck's symbols,

$$
q_{k}=q_{k-1}-m_{k}
$$

and

$$
m_{k}=m_{k-1}-n_{k} \operatorname{sgn} q_{k-1}
$$

are essential for the derivation of higher order corrections to the diffusion constant ${ }^{11}$. It is in order to emphasize that they are exact even at the quantum mechanical level, although originally found in the classical context ${ }^{11}$.

Note that above $m_{0}=m_{n}=0$. So far the discussions above are formally accurate. We assume now that the correlator, $\mathcal{D}_{0}\left(l, l^{\prime}\right)$ does not depend on the center-ofmass, namely $\mathcal{D}_{0}\left(l, l^{\prime}\right)=\mathcal{D}_{0}\left(l-l^{\prime}\right)$. This is supplemented by performing Fourier transformation for $\mathcal{D}_{0}\left(l-l^{\prime}\right)$ :

$$
\mathcal{D}_{0}\left(l-l^{\prime}\right)=\int \frac{d \varphi}{2 \pi} \mathcal{D}_{0}(\varphi) e^{i \varphi\left(l-l^{\prime}\right)}
$$

and imposing the boundary condition as (resulting from finite $\tau_{c}{ }^{11}$ )

$$
q_{0}=q_{r-1}=\varphi \rightarrow 0
$$

with $\varphi$ denoting the Fourier component. Then Fourier transforming $\Sigma\left(l-l^{\prime}\right)$ leads to

$$
\begin{aligned}
& \Sigma(\varphi)=e^{i \omega} J_{0}\left(\frac{2 K}{\hbar} \sin \frac{\hbar \varphi}{2}\right) \\
& +\sum_{r=2}^{\tau_{c}} e^{i \omega r} \sum_{n_{1} \neq 0} \sum_{n_{2}, \cdots, n_{r}} \sum_{m_{1}, m_{2}, \cdots, m_{r-1}} \sum_{q_{1}, \cdots, q_{r-2}} \prod_{k=1}^{r} \\
& \times J_{n_{1}}\left(\frac{2 K}{\hbar} \sin \frac{\hbar \varphi}{2}\right) J_{n_{r}}\left(\frac{2 K}{\hbar} \sin \frac{\hbar \varphi}{2}\right) \\
& \times J_{n_{k} \operatorname{sgn} q_{k-1}}\left(\frac{2 K}{\hbar}\left|\sin \frac{\hbar q_{k-1}}{2}\right|\right) .
\end{aligned}
$$

Note that above we suppressed the two Kroneck's symbols to simplify the expression. One should keep in mind that the sum over $m$ 's, $q$ 's and $n$ 's are restricted by the two "motion" equations, i.e., Eq. A8 and (A9).

Let us make the semiclassical approximation, i.e., $\left|k q_{k}\right| \ll 1$ in Eq. A12 and focus on the limit $K \varphi \ll 1$. The first term in the self-energy leads to the diffusion constant as $K^{2} / 4$, as discussed in Sec. IIIB The second term gives higher order oscillatory corrections. In fact, up to $(K \varphi)^{2}$, the sum is contributed by a particular series of $\left(q_{k}, m_{k}\right)(k=0,1,2, \cdots)$ (so-called Fourier paths $)^{11}$ as $(\varphi, 0) \rightarrow \pm(0,1) \cdots \pm(1,-1) \rightarrow(\varphi, 0)$. Here ... is shorthand of product of Bessel functions, which is an expansion in powers of $K^{-1 / 2}$. Since for $K \varphi \ll 1$, $J_{n}(K \varphi) \sim(K \varphi)^{n}$, therefore, $n_{1, r}= \pm 1$ is the only contribution to the order $(K \varphi)^{2}$. This then leads to $\Sigma(\varphi)$ as 11

$$
\begin{aligned}
& \Sigma(\varphi)=e^{i \omega} J_{0}(K \varphi) \\
& +\sum_{r=2}^{\tau_{c}} e^{i \omega r} \sum_{n_{1}, n_{r}= \pm 1} \sum_{n_{2}, \cdots, n_{r-1}} \sum_{m_{1}, m_{2}, \cdots, m_{r-1}} \sum_{q_{1}, \cdots, q_{r-2}} \prod_{k=1}^{r} \\
& \times J_{n_{1}}(K \varphi) J_{n_{r}}(K \varphi) J_{n_{k} \operatorname{sgn} q_{k-1}}\left(2 K\left|q_{k-1}\right|\right)
\end{aligned}
$$


In the limit $\omega \tau_{c} \ll 1, K \varphi \ll 1$, one recovers the diffuson Eq. (22) with the diffusion constant given by Eq. (23) up to $\mathcal{O}(1)$ (for $K \gg 1)^{11}$.

Proceeding along this line, we may reproduce Shepelansky's results for quantum diffusion constant of early evolution 31,56 . The basic observation is that if number of kicks is less than 4, then $q_{k}=1$. Based on Eq. (A12), this then implies that the results of Eq. (23) still holds except that $K$ is replaced by $2 K \sin (k / 2) / \hbar$.

\section{APPENDIX B: TWO RELATIONS RESULTING FROM TRS}

In this Appendix we show two exact relations reflecting TRS. In the first, for any $n>0$,

$$
\mathcal{D}_{0}\left(l, \theta ; l^{\prime}, \theta^{\prime} ; n\right)=\mathcal{D}_{0}\left(l^{\prime},-\theta^{\prime} ; l,-\theta ; n\right) .
$$

Proof. Use the mathematical deduction. For $n=1$,

$$
\begin{aligned}
& \mathcal{D}_{0}\left(l, \theta ; l^{\prime}, \theta^{\prime} ; 1\right) \\
= & \vec{P} \delta\left(l-l^{\prime}\right) \delta\left(\theta-\theta^{\prime}-l^{\prime}\right) \\
= & \delta\left(l-K \sin (\theta-l)-l^{\prime}\right) \delta\left(\theta-l-\theta^{\prime}-l^{\prime}\right) .
\end{aligned}
$$

On the other hand,

$$
\begin{aligned}
& \mathcal{D}_{0}\left(l^{\prime},-\theta^{\prime} ; l,-\theta ; 1\right) \\
= & \vec{P} \delta\left(l^{\prime}-l\right) \delta\left(-\theta^{\prime}+\theta-l\right) \\
= & \delta\left(l^{\prime}+K \sin \left(\theta^{\prime}+l^{\prime}\right)-l\right) \delta\left(-\theta^{\prime}-l^{\prime}+\theta-l\right) \\
= & \delta\left(l^{\prime}+K \sin (\theta-l)-l\right) \delta\left(\theta-l-\theta^{\prime}-l^{\prime}\right) .
\end{aligned}
$$

Comparing the last two lines of Eqs. (B2) and (B3), we immediately see that Eq. (B1) holds for $n=1$. Next we assume that Eq. (B1) holds for arbitrary $n=k>1$. Then for $n=k+1$, we obtain:

$$
\begin{aligned}
& \mathcal{D}_{0}\left(l^{\prime},-\theta^{\prime} ; l,-\theta ; k+1\right) \\
= & \vec{P} \mathcal{D}_{0}\left(l^{\prime},-\theta^{\prime} ; l,-\theta ; k\right) \\
= & \vec{P} \mathcal{D}_{0}\left(l, \theta ; l^{\prime}, \theta^{\prime} ; k\right) \\
\equiv & \mathcal{D}_{0}\left(l, \theta ; l^{\prime}, \theta^{\prime} ; k+1\right) .
\end{aligned}
$$

Thus, Eq. (B1) also holds for $n=k+1$. Q. E. D.

Now we turn to show the other relation. That is, if

$$
f\left(l, \theta ; l^{\prime}, \theta^{\prime}\right)=f\left(l^{\prime},-\theta^{\prime} ; l,-\theta\right),
$$

then

$$
\vec{P} f\left(l, \theta ; l^{\prime}, \theta^{\prime}\right)=f\left(l, \theta ; l^{\prime}, \theta^{\prime}\right) \overleftarrow{P}_{T}
$$

Proof.

$$
\begin{aligned}
& f\left(l, \theta ; l^{\prime}, \theta^{\prime}\right) \overleftarrow{P}_{T} \\
= & f\left(l, \theta ; l^{\prime}+K \sin \left(\theta^{\prime}+l^{\prime}\right), \theta^{\prime}+l^{\prime}\right) \\
= & f\left(l^{\prime}+K \sin \left(\theta^{\prime}+l^{\prime}\right),-\left(\theta^{\prime}+l^{\prime}\right) ; l,-\theta\right) \\
= & \vec{P} f\left(l^{\prime},-\theta^{\prime} ; l,-\theta\right) \\
= & \vec{P} f\left(l, \theta ; l^{\prime}, \theta^{\prime}\right)
\end{aligned}
$$

where we used the definition, Eq. (30), in the second line, Eq. (B5) in the third and fifth lines, and the definition, Eq. (17), in the fourth line. Q. E. D.

\section{APPENDIX C: DERIVATION OF RENORMALIZATION FACTOR OF DIFFUSIVE COOPERON}

In this Appendix, we derive the renormalization factor, Eq. (37), describing modification of the diffusive Cooperon due to the propagation through the Lyapunov region. We first analyze the asymptotic instability of a generic chaotic trajectory.

\section{Asymptotic instability}

By varying the equations of motion of the classical kicked rotor:

$$
\begin{aligned}
& \frac{d \theta}{d t}=l \\
& \frac{d l}{d t}=K \sin \theta \sum_{n} \delta(t-n)
\end{aligned}
$$

and defining the variables: $z \equiv \ln |\delta \theta|, \alpha \equiv \delta l / \delta \theta$, we obtain:

$$
\begin{aligned}
\frac{d z}{d t} & =\alpha, \\
\frac{d \alpha}{d t}+\alpha^{2} & =K \cos \theta \sum_{n} \delta(t-n),
\end{aligned}
$$

which describes the evolution of separation of two nearby trajectories along a reference trajectory, initiated from $\left(l_{0}, \theta_{0}\right)$. From Eq. C2 we see that $\alpha$ is a fast changing variable. That is, the dynamics of $\alpha$ introduces some classical time scale, beyond which the slow changing variable $-z$ is independent of initial $\alpha$. Let us further introduce the variables: $\alpha_{n}$-denoting $\alpha$ right after the $n$-th kicking, and $z_{n}$-denoting $z$ at the $n$-th kicking. Equivalently, we rewrite Eq. (C2) as

$$
\begin{aligned}
z_{n+1}-z_{n} & =\ln \left(1+\alpha_{n}\right), \\
\left.\alpha\right|_{n+1} & =\frac{1}{\left.\alpha^{-1}\right|_{n}+1}+K \cos \theta_{n+1} .
\end{aligned}
$$

For $n \gg 1,\left\langle z_{n}\right\rangle=\lambda n,\left\langle\left(z_{n}-\lambda n\right)^{2}\right\rangle=2 \lambda_{2} n$. Thus, the Lyapunov exponent $\lambda$ and its fluctuation $\lambda_{2}$, characterizing the long time instability are defined as

$$
\begin{aligned}
\lambda & =\lim _{n \rightarrow \infty} \lambda(n), \quad \lambda_{2}=\lim _{n \rightarrow \infty} \lambda_{2}(n), \\
\lambda(n) & =\frac{1}{n} \sum_{n^{\prime}=0}^{n-1} \ln \left(1+\left.\alpha\right|_{n^{\prime}}\right), \\
\lambda_{2}(n) & =\frac{1}{n}\left[\sum_{n^{\prime}=0}^{n-1} \ln \left(1+\left.\alpha\right|_{n^{\prime}}\right)-n \lambda\right]^{2},
\end{aligned}
$$


respectively. Note that at finite times, they are trajectory-dependent. In the limit $n \rightarrow \infty$, the time average is expected to be equivalent to the average over $\alpha$, as well as the initial conditions located in the stochastic region.

The estimation of $\lambda$ and $\lambda_{2}$ in the limit $K \gg 1$ may be made as follows based on the simple analysis above:

$$
\begin{aligned}
\lambda & \equiv\langle\ln |K \cos \theta|\rangle=\ln (K / 2) ; \\
\lambda_{2} & \equiv\left\langle\ln ^{2}|K \cos \theta|\right\rangle-\lambda^{2}=\zeta(3)-\ln ^{2} 2 \approx 0.82,
\end{aligned}
$$

where the angular brackets imply uniform averaging over the angle.

\section{Renormalization factor}

According to the definition of $n_{\mathrm{c}}, \mathrm{Eq}$. 34 it is the kick number when $z \approx 0$ with the logarithmic accuracy. That is,

$$
0=z_{0}+\sum_{j=0}^{n_{\mathrm{c}}-1} \ln \left(1+\left.\alpha\right|_{j}\right)
$$

equivalently,

$$
n_{\mathrm{c}}=-\frac{1}{\lambda\left(n_{\mathrm{c}}\right)}\left\{z_{0}+\sum_{j=0}^{n_{\mathrm{c}}-1}\left[\ln \left(1+\left.\alpha\right|_{j}\right)-\lambda\left(n_{\mathrm{c}}\right)\right]\right\} \text {. }
$$

Here $z_{0}$ is some function of the initial deviations $\left(\delta l_{0}, \delta \theta_{0}\right)$. The exact form of $z_{0}$ is unessential. For an estimate, we notice that in the limit $K \gg 1$ the evolution of $\delta \theta_{n}$ may be approximated as $\left(\delta \theta_{0}+\right.$ $\left.\delta l_{0} / K \cos \theta_{0}\right) \prod_{k=0}^{n-1} K \cos \theta_{k}$. Therefore,

$$
\begin{aligned}
z_{0} & =\ln \left|\delta \theta_{0}+\delta l_{0} / K \cos \theta_{0}\right| \\
& \simeq \ln \sqrt{\delta \theta_{0}^{2}+\delta l_{0}^{2} / K^{2}} .
\end{aligned}
$$

Substituting Eq. (C7) into $\exp \left(2 i \omega n_{\mathrm{c}}\right)$, we obtain:

$$
\begin{aligned}
e^{2 i \omega n_{\mathrm{c}}}= & \exp \left[-\frac{2 i \omega z_{0}}{\lambda\left(n_{\mathrm{c}}\right)}\right] \\
& \times \exp \left\{-\frac{2 i \omega}{\lambda\left(n_{c}\right)} \sum_{j=0}^{n_{\mathrm{c}}-1}\left[\ln \left(1+\left.\alpha\right|_{j}\right)-\lambda\left(n_{\mathrm{c}}\right)\right]\right\} \\
\approx & \exp \left[-\frac{2 i \omega z_{0}}{\lambda\left(n_{\mathrm{c}}\right)}\right] \exp \left[-\frac{2 \omega^{2} \lambda_{2}\left(n_{\mathrm{c}}\right) z_{0}}{\lambda^{2}\left(n_{\mathrm{c}}\right)}\right]
\end{aligned}
$$

where in the second line we use the fact that $\omega \lambda_{2}\left(n_{\mathrm{c}}\right) n_{\mathrm{c}} / \lambda^{2}\left(n_{\mathrm{c}}\right) \ll 1$. In the limit $n_{\mathrm{c}} \gg 1, \lambda\left(n_{\mathrm{c}}\right) \rightarrow$ $\lambda, \lambda_{2}\left(n_{\mathrm{c}}\right) \rightarrow \lambda_{2}$. Moreover, from Eq. (C7) we see that $n_{\mathrm{c}} \rightarrow t^{\mathrm{C}} \equiv-z_{0} / \lambda$. Thus, Eq. (C9) is reduced to Eq. (37). (a)

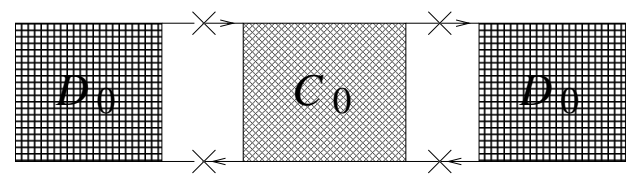

(b)

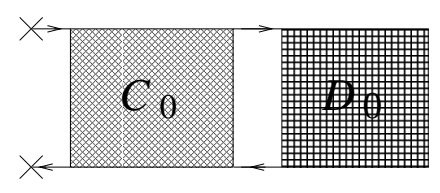

(c)

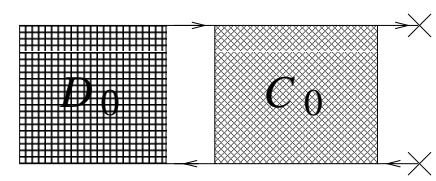

FIG. 9: Diagrams that lead to the interaction vertex with the particle conservation law respected: (a) $\delta \hat{\mathcal{D}}_{1}$, (b) $\delta \hat{\mathcal{D}}_{2}$, and (c) $\delta \hat{\mathcal{D}}_{3}$.

\section{APPENDIX D: THE EXACT INTERACTION VERTEX}

In this Appendix we discuss the exact interaction vertex appearing in the one-loop calculation. The general diagram, sketched in Fig. 5] may be categorized into three classes, Fig. 9] a-c. In order to make the formula compact, let us write them down in the operator representation as:

$$
\begin{aligned}
\delta \hat{\mathcal{D}}_{1} & =\left(e^{i \omega} \hat{\mathcal{P}} \hat{\mathcal{D}}\right) \hat{\mathrm{C}}\left(e^{i \omega} \hat{\mathcal{P}} \hat{\mathcal{D}}\right) \\
\delta \hat{\mathcal{D}}_{2} & =\hat{\mathcal{P}}_{V} \hat{\mathrm{C}} \hat{\mathcal{D}} \\
\delta \hat{\mathcal{D}}_{3} & =\hat{\mathcal{D}} \hat{\mathrm{C}} \hat{\mathcal{P}}_{V} .
\end{aligned}
$$

We then insert the identity :

$$
e^{i \omega} \hat{\mathcal{P}} \equiv 1+\left(e^{i \omega} \hat{\mathcal{P}}-1\right)
$$

into Eq. (D1) and rewrite $\delta \hat{\mathcal{D}}_{1}$ as

$$
\delta \hat{\mathcal{D}}_{1} \equiv \hat{\mathcal{D}} \hat{\mathbf{C}} \hat{\mathcal{D}}+\delta \hat{\mathcal{D}}_{1}^{\prime}+\delta \hat{\mathcal{D}}_{4}
$$

with

$$
\begin{aligned}
& \delta \hat{\mathcal{D}}_{1}^{\prime}=\left[\left(e^{i \omega} \hat{\mathcal{P}}-1\right) \hat{\mathcal{D}}\right] \hat{\mathrm{C}} \hat{\mathcal{D}}+\hat{\mathcal{D}} \hat{\mathrm{C}}\left[\left(e^{i \omega} \hat{\mathcal{P}}-1\right) \hat{\mathcal{D}}\right] \\
& \delta \hat{\mathcal{D}}_{4}=\left[\left(e^{i \omega} \hat{\mathcal{P}}-1\right) \hat{\mathcal{D}}\right] \hat{\mathrm{C}}\left[\left(e^{i \omega} \hat{\mathcal{P}}-1\right) \hat{\mathcal{D}}\right] .
\end{aligned}
$$

For the first term in Eq. (D5), it was proved by Altland ${ }^{18}$ that it vanishes in the quantum limit. In the next section, we show that this term is indeed pure classical, and does not contribute to the quantum interference correction. By the same token we show that the loop expansion does not violate the particle conservation law. That is the form of the interaction vertex implies that the quantum corrections may be reduced to the renormalization of the diffusion coefficient. 


\section{Semiclassical analysis on $\hat{\mathcal{D}} \hat{\mathbf{C}} \hat{\mathcal{D}}$}

Under appropriate approximation, it was proved that $\hat{\mathcal{D}} \hat{\mathbf{C}} \hat{\mathcal{D}}$ is included into classical diffusive propagator ${ }^{18}$. Thus it does not violate diffusion equation. Since we are interested in the dynamics involving Ehrenfest time, the diffusive propagator can not serve as a starting point of the formalism developed here. A natural question is whether this conclusion may still be applicable. Although it remains a challenge to prove at the accurate level, here we present a physical interpretation. We conclude that this term is pure classical, included in the exact classical propagator-solution of the FPR equation.

For quantitative discussions below, let us denote the angular momenta and angles, appearing in the retarded/advanced propagator lines of Cooperon as $l_{1 \pm}, l_{2 \pm}, \cdots, l_{n \pm}$ and $\theta_{1 \pm}, \theta_{2 \pm}, \cdots, \theta_{(n-1) \pm}$ following the forward time direction of the retarded line (see Fig. [5] for notations). Counting from the most left hand side of the Cooperon and following the backward time direction of the retarded line, we denote those as $l_{0 \pm}, l_{-1 \pm}, l_{-2 \pm}, \cdots$, and $\theta_{0 \pm}, \theta_{-1 \pm}, \theta_{-2 \pm}, \cdots$. Semiclassically, only the constraint below

$$
\left|\theta_{1+}+\theta_{(n-1)-}\right| \sim\left|\theta_{2+}+\theta_{(n-2)-}\right| \cdots\left|\theta_{(n-1)+}+\theta_{1-}\right| \lesssim k
$$

is imposed.

According to the exact Eq. A8, at the boundary of Cooperon and (left) Diffuson,

$$
\left(\theta_{1+}-\theta_{1-}\right)=\left(\theta_{0+}-\theta_{0-}\right)-\left(l_{1+}-\theta_{1-}\right) .
$$

On the other hand, Eq. (D7) does not impose any constraints on $\theta_{1+}-\theta_{1-}$. If it is order $k$, the usual Wigner transform may be performed (see Eq. (14)). That is to say, the left-most kicks of the Cooperon may be incorporated into the left Diffuson.

This discussions remain applicable for all the successive pairs of kicks of the Cooperon, until we meet some $k>1$, where $\theta_{k+}-\theta_{k-} \sim 1$-a typical feature of the Cooperon. This is equivalent to the validity of Eq. (D8) with the right hand side of order 1 . In fact, $\left(\theta_{0+}-\theta_{0-}\right)$ and $\left(l_{1+}-\theta_{1-}\right)$ develop from the left (classical) Diffuson-following Eqs. (A8) and (A9). In the Diffuson side, $\left(\theta_{k+}-\theta_{k-}\right)(k=-1,-2, \cdots),\left(l_{k+}-l_{k-}\right)(k=$ $0,-1,-2, \cdots)$ are of order $k$. Moreover, any evolution can last at most finite classical correlation time $\tau_{c}$. Thus, $\left(\theta_{1+}-\theta_{1-}\right)$ must be of order $k$. Thus, Eq. (D8) can not be satisfied if $\left(\theta_{1+}-\theta_{1-}\right) \sim \mathcal{O}(1)$.

Thus we may conclude that $\hat{\mathcal{D}} \hat{\mathbf{C}} \hat{\mathcal{D}}$ is pure classical. It characterizes the classical probability of trajectories with a peculiar feature. Since $\left|\theta_{1-}+\theta_{n+}\right| \lesssim \hbar \ll 1$, $\theta_{1+} \sim \theta_{1-} \sim-\theta_{n+}$, the trajectory switches its angle to the direction (almost) opposite to the initial.

\section{A cancellation mechanism}

Having the general expression, Eq. (52) at hand, we turn to show that $\delta \hat{\mathcal{D}}_{1}^{\prime}, \delta \hat{\mathcal{D}}_{2}, \delta \hat{\mathcal{D}}_{3}$ cancel each other at the semiclassical level, namely:

$$
\delta \mathcal{D}_{1}^{\prime}+\delta \mathcal{D}_{2}+\delta \mathcal{D}_{3} \equiv 0
$$

Indeed, employing identity, Eq. (B6), we obtain:

$$
\begin{aligned}
& \delta \mathcal{D}_{1}^{\prime}\left(l, \theta ; l^{\prime}, \theta^{\prime}\right) \\
= & \hat{\mathcal{V}}\left\{\mathcal { D } _ { 0 } ( l , \theta ; l ^ { \prime \prime } + \frac { \delta l _ { 2 } } { 2 } , \theta ^ { \prime \prime } + \frac { \delta \theta _ { 1 } } { 2 } ) \left[\left(e^{i \omega} \overleftarrow{P}_{T}-1\right)\right.\right. \\
& \left.\left.+\left(e^{i \omega} \vec{P}-1\right)\right] \mathcal{D}_{0}\left(l^{\prime \prime}-\frac{\delta l_{2}}{2},-\theta^{\prime \prime}+\frac{\delta \theta_{1}}{2} ; l^{\prime}, \theta^{\prime}\right)\right\}, \\
& \delta \mathcal{D}_{2}\left(l, \theta ; l^{\prime}, \theta^{\prime}\right) \\
= & \hat{\mathcal{V}}\left\{2 \pi k \delta\left[l-\left(l^{\prime \prime}+\frac{\delta l_{2}}{2}\right)\right] \delta\left[\theta-\left(\theta^{\prime \prime}+\frac{\delta \theta_{1}}{2}\right)-l\right]\right. \\
& \left.\times \mathcal{D}_{0}\left(l^{\prime \prime}-\frac{\delta l_{2}}{2},-\theta^{\prime \prime}+\frac{\delta \theta_{1}}{2} ; l^{\prime}, \theta^{\prime}\right)\right\}
\end{aligned}
$$

and

$$
\begin{array}{r}
\delta \mathcal{D}_{3}\left(l, \theta ; l^{\prime}, \theta^{\prime}\right)=\hat{\mathcal{V}}\left[\mathcal{D}_{0}\left(l, \theta ; l^{\prime \prime}+\frac{\delta l_{2}}{2}, \theta^{\prime \prime}+\frac{\delta \theta_{1}}{2}\right)\right. \\
\left.\times 2 \pi \hbar \delta\left(l^{\prime \prime}-\frac{\delta l_{2}}{2}-l^{\prime}\right) \delta\left(-\theta^{\prime \prime}+\frac{\delta \theta_{1}}{2}-\theta^{\prime}-l^{\prime \prime}-\frac{\delta l_{2}}{2}\right)\right] .
\end{array}
$$

Since $\mathcal{D}_{0}$ is the solution of FPR equation, i.e., Eq. (16), these three terms cancel each other. Therefore we find that only $\delta \mathcal{D}_{4}$ leads to the non-vanishing one-loop correction to the classical density-density correlator.

\section{APPENDIX E: ALEINER-LARKIN REGULARIZATION IN KICKED ROTOR}

It is easy to see that in the classical limit: $\hbar \rightarrow 0$, the functional form of $\delta \mathcal{D}_{1}^{\prime}$ and $\delta \mathcal{D}_{2,3}$, i.e., Eqs. (D10), (D11) and (D12) are identical to what have been found for classical Lorentz gas (leaving the feature of standard map aside). These terms cancel each other, leading to the absence of the weak localization correction. Remarkably, this cancellation still holds, even if the initial minimal wave packet is taken into account. On the other hand, the weak localization correction does exist, given by the exact vertex $\delta \mathcal{D}_{4}$, which is absent in the expansion of ballistic supersymmetric $\sigma$ model (without a regularizer). In this way, one may wonder that an appropriate regularization may lead to a physical description of weak localization in semiclassical chaotic systems. This, indeed, was developed by Aleiner and Larkin for ballistic electronic problems 27.58 . However, an important issue remains open whether and to what extent the physical 
results depend on the regularizer, rather than on the intrinsic quantum nature of the problem. The exact interaction vertex may serve as a testing ground of this regularization.

In fact, the regularization introduced in Refs. 27,58 is also applicable for QKR. To this end we try to mimic the "Born impurity" by modifying the (quantum) free rotation operator $\hat{\mathcal{P}}_{V}$ in the following way:

$$
\hat{\mathcal{P}}_{V} \rightarrow \hat{\mathcal{P}}_{V} \exp \left(\frac{i \delta \hat{S}}{\hbar}\right)
$$

Here $\delta \hat{S}$ is some stationary random perturbation that commutes with $\hat{\mathcal{P}}_{V}$, i.e., $\left[\delta \hat{S}, \hat{\mathcal{P}}_{V}\right]=0$. Moreover, we assume that it is short-ranged correlated in the angular momentum space:

$$
\left\langle\partial_{l} \delta S_{l} \partial_{l^{\prime}} \delta S_{l^{\prime}}\right\rangle=\frac{2}{\tau_{q}} \delta_{l, l^{\prime}}
$$

In the classical limit, this additional stationary random perturbations leads to the modification of the standard mapping in the following way

$$
\begin{aligned}
l_{n+1} & =l_{n}+K \sin \theta_{n}, \\
\theta_{n+1} & =\theta_{n}+l_{n+1}+\partial_{l_{n+1}} \delta S_{l_{n+1}} .
\end{aligned}
$$

Then following the same procedure as in Ref. 27, we find the one-loop correction to the density-density correlator to be

$$
\begin{aligned}
& \delta \mathcal{D}\left(l, \theta ; l^{\prime}, \theta^{\prime}\right) \\
= & \frac{2}{\tau_{q}} \int \frac{d l_{1} d \theta_{1}}{2 \pi \hbar} \mathcal{C}\left(l_{l}, \theta_{1} ; l_{1},-\theta_{1}\right) \\
\times & {\left[\frac{\partial}{\partial \theta_{1}} e^{i \omega} \vec{P} \mathcal{D}\left(l, \theta ; l_{1}, \theta_{1}\right)\right]\left[\frac{\partial}{\partial \theta_{1}} e^{i \omega} \vec{P} \mathcal{D}\left(l_{1},-\theta_{1} ; l^{\prime}, \theta^{\prime}\right)\right] }
\end{aligned}
$$

1 D. M. Basko, M. A. Skvortsov, and V. E. Kravtsov, Phys. Rev. Lett. 90, 096801 (2003); V. E. Kravtsov, cond-mat/0312316

2 F. L. Moore et al., Phys. Rev. Lett. 75, 4598 (1995); C. F. Bharucha et al., Phys. Rev. E 60,3881 (1999).

3 M. G. Raizen, Adv. At., Mol., Opt. Phys. 41, 43 (1999).

${ }^{4}$ C. Zhang, J. Liu, M. Raizen, and Q. Niu, Phys. Rev. Lett. 92, 054101 (2004).

${ }^{5}$ O. A. Starykh, P. R. J. Jacquod, E. E. Narimanov, and A. D. Stone, Phys. Rev. E 62, 2078 (2000).

${ }^{6}$ G. Casati, B. V. Chirikov, J. Ford, and F. M. Izrailev, in Stochastic Behavior of Classical and Quantum Hamiltonian Systems, Lecture Notes in Physics 93, edited by G. Casati and J. Ford, 334 (Springer, New York 1979).

7 B. V. Chirikov, F. M. Izrailev, and D. L. Shepelyansky, Sov. Sci. Rev. Sec. C 2, 209 (1981).

8 H. Ammann et al., Phys. Rev. Lett. 80, 4111 (1998).

9 B. V. Chirikov, Phys. Rep. 52, 263 (1979).

10 A. L. Lichtenberg and M. A. Lieberman, Regular and Chaotic Dynamics (Springer-Verlag, Berlin 1991). with the regularized Diffuson $\mathcal{D}$ and the Cooperon $\mathcal{C}$ satisfying the following equation:

$$
\begin{aligned}
& {\left[1-\left(1+\frac{1}{\tau_{q}} \frac{\partial^{2}}{\partial \theta^{2}}\right) e^{i \omega} \vec{P}\right]\left\{\begin{array}{l}
\mathcal{D}\left(l, \theta ; l^{\prime}, \theta^{\prime}\right) \\
\mathcal{C}\left(l, \theta ; l^{\prime}, \theta^{\prime}\right)
\end{array}\right\} } \\
= & 2 \pi \hbar \delta\left(l-l^{\prime}\right) \delta\left(\theta-\theta^{\prime}\right) .
\end{aligned}
$$

Eqs. (E4) and (E5) are fully analogous to those found for ballistic electronic systems. In Eq. (E5), the regularizer of FRP equation mimics the spread of minimal wave packet arising from intrinsic quantum diffractions. Originally, it was expected 27 that the only physical effect arising from this regularizer is to determine the Ehrenfest time since it smears the sharp classical propagator. Indeed, one may further follow the procedure of Ref. 27 to calculate the weak-localization correction to the diffusion constant. As a result, the functional form of Eq. (82) is reproduced with $t_{E}$ acquiring explicit $\tau_{q^{-}}$ dependence 27 . At $\left(\lambda \tau_{q}\right)^{-1} \sim \hbar / K$, the result thereby obtained are identical to Eq. (82). This reflects a basic belief previously anticipated $27,28,44$. That is, a physical regularizer strength must match the minimal quantum wave packet.
11 A. B. Rechester and R. B. White, Phys. Rev. Lett. 44, 1586 (1980); A. B. Rechester, M. N. Rosenbluth, and R. B. White, Phys. Rev. A 23, 2664 (1981).

12 M. Khodas and S. Fishman, Phys. Rev. Lett. 84, 2837 (2000); Erratum, ibid. 84, 5918 (2000); M. Khodas, S. Fishman, and O. Agam, Phys. Rev. E 62, 4769 (2000).

13 S. Fishman, D. R. Grempel, and R. E. Prange, Phys. Rev. Lett. 49, 509 (1982).

14 S. Fishman, D. R. Grempel, and R. E. Prange, Phys. Rev. A 29, 1639 (1984).

15 R. Blümel and U. Smilansky, Phys. Rev. Lett. 69, 217 (1992).

16 P. A. Lee and T. V. Ramakrishnan, Rev. Mod. Phys. 57, 287 (1985).

17 L. P. Gorkov, A. I. Larkin, and D. E. Khmelnitskii, Pis'ma Zh. Eksp. Teor. Fiz. 30, 248 (1979) [JETP Lett. 30 , 248 (1979)].

18 A. Altland, Phys. Rev. Lett. 71, 69 (1993).

19 A. Altland and M. R. Zirnbauer, Phys. Rev. Lett. 77, 4536 (1996). 
${ }^{20}$ K. Efetov, Supersymmetry in disorder and chaos (Cambridge University Press, UK 1997).

21 A. I. Larkin and Yu. N. Ovchinnikov, Zh. Eksp. Teor. Fiz. 55, 2262 (1968) [Sov. Phys. JETP 28, 1200 (1969)].

22 G. P. Berman and G. M. Zaslavsky, Physica A 91, 450 (1978); G. M. Zaslavsky, Phys. Rep. 80, 157 (1981).

23 For a review, see F. M. Izrailev, Phys. Rep. 196, 299 (1990).

$24 \mathrm{Ph}$. Jacquod and E. V. Sukhorukov, Phys. Rev. Lett. 92, 116801 (2004); J. Tworzydlo, A. Tajic, and C. W. J. Beenakker, Phys. Rev. B 69, 165318 (2004), cond-mat/0405122

25 M. C. Goorden, Ph. Jacquod, and C. W. J. Beenakker, Phys. Rev. B 68, 220501(R) (2003).

26 J. Tworzydlo, A. Tajic, H. Schomerus, and C. W. J. Beenakker, Phys. Rev. B 68, 115313 (2003); P. G. Silvestrov, M. C. Goorden, and C. W. J. Beenakker, Phys. Rev. Lett. 90, 116801 (2003).

27 I. L Aleiner and A. I. Larkin, Phys. Rev. B 54, 14423 (1996).

28 M. G. Vavilov and A. I. Larkin, Phys. Rev. B 67, 115335 (2003).

29 C. Tian, A. Kamenev, and A. Larkin, Phys. Rev. Lett. 93, 124101 (2004).

30 J. Müler and A. Altland, nlin.CD/0411055

31 D. L. Shepelyansky, Physica D 28, 103 (1987).

32 D. A. Steck, V. Milner, W. H. Oskay, and M. G. Raizen, Phys. Rev. E 62, 3461 (2000).

33 P. H. Jones et. al., physics/0405046 P. H. Jones et. al., quant-ph/0309149

34 S. Montangero, A. Romito, G. Benenti, and R. Fazio, cond-mat/0407274

35 A. Isacsson, L. Y. Gorelik, R. I. Shekhter, Y. M. Galperin, and M. Jonson, Phys. Rev. Lett. 89, 277002 (2002); L. Y. Gorelik, A. Isacsson, Y. M. Galperin, R. I. Shekhter, and M. Jonson, Nature 411,454 (2001).

36 S. Hikami, Phys. Rev. B 24, 2671 (1981).

37 M. R. Zirnbauer, in Supersymmetry and Trace Formulae, Chaos and Disorder, edited by I. V. Lerner, J. P. Keating and D. E. Khmelnitskii (Kluwer Academic/Plenum, New York 1999).

38 See, e.g., G. M. Zaslavsky, M. Edelman, and B. A. Niyazov, Chaos 7, 159 (1997).

39 F. M. Izrailev, Phys. Rev. Lett. 56, 541 (1986).

40 M. Thaha, R. Blümel, and U. Smilansky, Phys. Rev. E 48, 1764 (1993).
41 T. Tworzydlo, A. Tajic, and C. W. J. Beenakker, cond-mat/0405122

42 R. A. Smith, I. V. Lerner, and B. L. Altshuler, Phys. Rev. B 58, 10343 (1998)

43 R. S. Whitney, I. V. Lerner, and R. A. Smith, Waves Random Media 9, 179 (1999).

44 C. Tian and A. I. Larkin, Phys. Rev. B 70, 035305 (2004).

45 For a review see e.g. S. Chu, Science 253, 861 (1991); W. D. Phillips, Rev. Mod. Phys. 70, 721 (1998); C. E. Wieman, D. E. Pritchard, and D. J. Wineland, Rev. Mod. Phys. 71, S253 (1999).

46 R. Graham, M. Schlautmann, and P. Zoller, Phys. Rev. A 45, R19 (1992).

47 F. L. Moore et al., Phys. Rev. Lett. 73, 2974 (1994); J. C. Robinson et al., Phys. Rev. Lett. 74, 3963 (1995).

48 In the conventional QKR is periodic, i.e. $\Psi(\theta+2 \pi)=\Psi(\theta)$, leading to the discrete angular momentum: $l=n k$ with $n$ integer. Periodically kicked cold atomic gases differs from it, with the boundary condition replaced by: $\Psi(\theta+2 \pi)=$ $\Psi(\theta) e^{i \theta l_{0}}$, where $l_{0} \in[0,1]$ is a fractional part of an atom's momentum in units $2 \hbar k_{L}$. It may be eliminated by the gauge transformation that introduces an Aharonov-Bohm flux into the kinetic energy $\left(\hat{l}-l_{0}\right)^{2} / 2$. Such flux does not affect dynamical localization, though quantum resonances ${ }^{23}$ is eliminated by averaging over $l_{0}$.

49 C. Tian, A. Kamenev, and A. I. Larkin, Bull. Am. Phys. Soc. 49, 482 (2004).

50 E. Chow, P. Delsing, and D. B. Haviland, Phys. Rev. Lett. 81, 204 (1998).

51 D. B. Haviland and P. Delsing, Phys. Rev. B 54, R6857 (1996).

52 M. Watanabe and D. B. Haviland, in Studies of High Temperature Superconductors (Advances in Research and Applications), Vol. 43, edited by A. Narlikar (Nova Science Publishers, New York, 2002).

53 D. Cohen, Phys. Rev. A 44, 2292 (1991).

${ }^{54}$ V. Milner et. al., Phys. Rev. E 61, 7223 (2000).

55 B. G. Klappauf et. al., Phys. Rev. Lett. 81, 1203 (1998).

56 A. J. Daley and A. S. Parkins, Phys. Rev. E 66, 056210 (2002); G. Duffy et. al., cond-mat/0401346

57 K. B. Efetov, G. Schwiete, and K. Takahashi, Phys. Rev. Lett. 92, 026807 (2004).

58 I. L. Aleiner and A. I. Larkin, Phys. Rev. E 55, R1243 (1997). 